\title{
DIMENSIONS OF LOCALLY AND ASYMPTOTICALLY SELF-SIMILAR SPACES
}

\author{
S. BUYALO AND N. LEBEDEVA
}

To dear Viktor Abramovich Zalgaller, on the occasion of his 85th birthday

\begin{abstract}
Two results are obtained, in a sense dual to each other. First, the capacity dimension of every compact, locally self-similar metric space coincides with the topological dimension, and second, a metric space asymptotically similar to its compact subspace has asymptotic dimension equal to the topological dimension of the subspace. As an application of the first result, the following Gromov conjecture is proved: the asymptotic dimension of every hyperbolic group $G$ equals the topological dimension of its boundary at infinity plus 1 , asdim $G=\operatorname{dim} \partial_{\infty} G+1$. As an application of the second result, we construct Pontryagin surfaces for the asymptotic dimension; in particular, these surfaces are examples of metric spaces $X, Y$ with $\operatorname{asdim}(X \times Y)<\operatorname{asdim} X+\operatorname{asdim} Y$. Other applications are also given.
\end{abstract}

\section{$\S 1$. INTRODUCTION}

We say that a map $f: Z \rightarrow Z^{\prime}$ between metric spaces is quasihomothetic with coefficient $R>0$ if we have

$$
R\left|z z^{\prime}\right| / \lambda \leq\left|f(z) f\left(z^{\prime}\right)\right| \leq \lambda R\left|z z^{\prime}\right|
$$

for some $\lambda \geq 1$ and for all $z, z^{\prime} \in Z$. In this case, we also say that $f$ is $\lambda$-quasihomothetic with coefficient $R$.

A metric space $Z$ is locally similar to a metric space $Y$ if there is $\lambda \geq 1$ such that for every sufficiently large $R>1$ and every $A \subset Z$ with $\operatorname{diam} A \leq \Lambda_{0} / R$, where $\Lambda_{0}=$ $\min \{1, \operatorname{diam} Y / \lambda\}$, there is a $\lambda$-quasihomothetic map $f: A \rightarrow Y$ with coefficient $R$ (note that the condition diam $A \leq \Lambda_{0} / R$ implies $\left.\operatorname{diam} f(A) \leq \operatorname{diam} Y\right)$. If a metric space $Z$ is locally similar to itself, then we say that $Z$ is locally self-similar.

The notion of the capacity $\operatorname{dimension} \operatorname{cdim} Z$ of a metric space $Z$ was introduced in [Bu1] and turned out to be useful in many questions; see [Bu2]. The capacity dimension is greater than or equal to the topological dimension, $\operatorname{dim} Z \leq \operatorname{cdim} Z$ for every metric space $Z$, and it is important to know for what spaces equality occurs. Our first main result states the following.

Theorem 1.1. Assume that a metric space $Z$ is locally similar to a compact metric space $Y$. Then $\operatorname{cdim} Z<\infty$ and $\operatorname{cdim} Z \leq \operatorname{dim} Y$.

Corollary 1.2. The capacity dimension of every compact, locally self-similar metric space $Z$ is finite and coincides with its topological dimension, $\operatorname{cdim} Z=\operatorname{dim} Z$.

2000 Mathematics Subject Classification. Primary 51F99, 55M10.

Key words and phrases. Asymptotic dimension, self-similar spaces.

Both authors were supported by RFBR (grant 05-01-00939) and by grant NSH-1914.2003.1. 
On the other hand, we also prove a proposition (see Proposition 4.1) that makes it possible to construct examples of compact metric spaces for which the capacity dimension is arbitrarily larger than the topological dimension.

Now, consider the dual situation (in a sense). A metric space $X$ is asymptotically similar to a metric space $Y$ if there are $\Lambda_{0}, \lambda \geq 1$ such that for every sufficiently large $R>1$ and every $A \subset X$ with $\operatorname{diam} A \leq R / \Lambda_{0}$ there is a $\lambda$-quasihomothetic map $f: Y \rightarrow$ $X$ with coefficient $R$ whose image contains an isometric copy $A^{\prime} \subset X$ of $A, A^{\prime} \subset f(Y)$. If a metric space $X$ is asymptotically similar to a bounded subset, then we say that $X$ is asymptotically self-similar. Taking a copy $A^{\prime}$ instead of $A$ provides an additional flexibility of this definition, which is necessary for applications; see Subsection 7.4.

We recall the well-established notion of the asymptotic dimension, asdim, in Subsection 2.1.

Our second main result is as follows.

Theorem 1.3. Assume that a metric space $X$ is asymptotically similar to a compact metric space $Y$. Then both dimensions asdim $X$ and $\operatorname{dim} Y$ are finite and coincide, $\operatorname{asdim} X=\operatorname{dim} Y$.

As applications of Theorem 1.1, we obtain the following results.

Theorem 1.4. The capacity dimension of the boundary at infinity of any hyperbolic group $G$ (taken with any visual metric) coincides with the topological dimension of $G$, $\operatorname{cdim} \partial_{\infty} G=\operatorname{dim} \partial_{\infty} G$.

Combined together with the main result of [Bu1], Theorem 1.4 leads to the following result, which proves Gromov's conjecture; see [Gr, 1. $\left.\mathrm{E}_{1}^{\prime}\right]$.

Theorem 1.5. The asymptotic dimension of any hyperbolic group $G$ is equal to the topological dimension of its boundary at infinity plus 1 ,

$$
\operatorname{asdim} G=\operatorname{dim} \partial_{\infty} G+1 .
$$

Another application of Theorem 1.4 is the following embedding result, obtained from the main result of $[\mathrm{Bu} 2]$.

Theorem 1.6. Every hyperbolic group $G$ admits a quasi-isometric embedding $G \rightarrow T_{1} \times$ $\cdots \times T_{n}$ into the $n$-fold product of simplicial metric trees $T_{1}, \ldots, T_{n}$ with $n=\operatorname{dim} \partial_{\infty} G+1$.

The group structure plays no role in the proof of Theorems 1.4-1.6. Actually, we prove more general Theorems 6.3, 6.4, and 6.6; the statements above are chosen for the simplicity of their formulation.

Theorem 6.3 has applications also to nonembedding results, which are discussed in Subsection 6.3; see Theorem 6.7. Using Corollary 1.2, we give examples of strict inequality in the product theorem for the capacity dimension. These are famous Pontryagin surfaces, the self-similar construction of which is discussed in $\S 7$; see Theorem 7.1. Finally, we construct metric spaces asymptotically similar to self-similar Pontryagin surfaces. As a corollary to Theorem 1.3, we give examples of strict inequality in the product theorem for the asymptotic dimension; that is, we construct metric spaces $X, Y$ with

$$
\operatorname{asdim}(X \times Y)<\operatorname{asdim} X+\operatorname{asdim} Y
$$

(see Corollary 7.7).

\section{§2. Preliminaries}

Here, we recall some notions and facts necessary for the paper.

Let $Z$ be a metric space. For $U, U^{\prime} \subset Z$ we denote by $\operatorname{dist}\left(U, U^{\prime}\right)$ the distance between $U$ and $U^{\prime}, \operatorname{dist}\left(U, U^{\prime}\right)=\inf \left\{\left|u u^{\prime}\right|: u \in U, u^{\prime} \in U^{\prime}\right\}$, where $\left|u u^{\prime}\right|$ is the distance 
between $u$ and $u^{\prime}$. For $r>0$ we denote by $B_{r}(U)$ the open $r$-neighborhood of $U$, $B_{r}(U)=\{z \in Z: \operatorname{dist}(z, U)<r\}$, and by $\bar{B}_{r}(U)$ the closed $r$-neighborhood of $U$, $\bar{B}_{r}(U)=\{z \in Z$ : $\operatorname{dist}(z, U) \leq r\}$. We extend this notation to all real $r$ by putting $B_{r}(U)=U$ for $r=0$, and defining $B_{r}(U)$ for $r<0$ as the complement of the closed $|r|$-neighborhood of $Z \backslash U, B_{r}(U)=Z \backslash \bar{B}_{|r|}(Z \backslash U)$.

Given a family $\mathcal{U}$ of subsets in a metric space $Z$, we define $\operatorname{mesh}(\mathcal{U})=\sup \{\operatorname{diam} U$ : $U \in \mathcal{U}\}$. The multiplicity $m(\mathcal{U})$ of $\mathcal{U}$ is the maximal number of members of $\mathcal{U}$ with nonempty intersection. We say that a family $\mathcal{U}$ is disjoint if $m(\mathcal{U})=1$.

A family $\mathcal{U}$ is called a covering of $Z$ if $\bigcup\{U: U \in \mathcal{U}\}=Z$. A covering $\mathcal{U}$ is said to be colored if it is the union of $m \geq 1$ disjoint families, $\mathcal{U}=\bigcup_{a \in A} \mathcal{U}^{a},|A|=m$. In this case we also say that $\mathcal{U}$ is $m$-colored. Clearly, the multiplicity of an $m$-colored covering is at most $m$.

Let $\mathcal{U}$ be a family of open subsets in a metric space $Z$ that covers $A \subset Z$. For $z \in A$, let

$$
L(\mathcal{U}, z)=\sup \{\operatorname{dist}(z, Z \backslash U): U \in \mathcal{U}\}
$$

be the Lebesgue number of $\mathcal{U}$ at $z$, and let $L(\mathcal{U})=\inf _{z \in A} L(\mathcal{U}, z)$ be the Lebesgue number of the covering $\mathcal{U}$ of $A$. For every $z \in A$, the open ball $B_{r}(z) \subset Z$ of radius $r=L(\mathcal{U})$ centered at $z$ is contained in some member of the covering $\mathcal{U}$.

We shall use the following obvious fact (see e.g. [Bu1]).

Lemma 2.1. Let $\mathcal{U}$ be an open covering of $A \subset Z$ with $L(\mathcal{U})>0$. Then for every $s \in(0, L(\mathcal{U}))$ the family $\mathcal{U}_{-s}=B_{-s}(\mathcal{U})$ is still an open covering of $A$.

2.1. Definitions of the capacity dimension and the asymptotic dimension. There are several equivalent definitions of the capacity dimension; see [Bu1]. In this paper, we use the following two.

(i) The capacity $\operatorname{dimension} \operatorname{cdim}(Z)$ of a metric space $Z$ is the minimal integer $m \geq 0$ with the following property: there is a constant $\delta \in(0,1)$ such that for every sufficiently small $\tau>0$ there exists an $(m+1)$-colored open covering $\mathcal{U}$ of $Z$ with $\operatorname{mesh}(\mathcal{U}) \leq \tau$ and $L(\mathcal{U}) \geq \delta \tau$.

(ii) The capacity dimension $\operatorname{cdim}(Z)$ of a metric space $Z$ is the minimal integer $m \geq 0$ with the following property: there is a constant $\delta \in(0,1)$ such that for every sufficiently small $\tau>0$ there exists an open covering $\mathcal{U}$ of $Z$ with multiplicity $m(\mathcal{U}) \leq m+1$ and such that $\operatorname{mesh}(\mathcal{U}) \leq \tau$ and $L(\mathcal{U}) \geq \delta \tau$.

The asymptotic dimension is a quasi-isometry invariant of a metric space; it was introduced in $[\mathrm{Gr}]$. There are also several equivalent definitions, see [Gr], [BD], and we use the following one. The asymptotic dimension of a metric space $X$ is the minimal integer asdim $X=n$ such that for every positive $d$ there is an open covering $\mathcal{U}$ of $X$ with $m(\mathcal{U}) \leq n+1, \operatorname{mesh}(\mathcal{U})<\infty$, and $L(\mathcal{U}) \geq d$.

The following notion turns out to be useful for our purposes. It is called the Higson property in [DZ, §4] and the asymptotic dimension of linear type in [Ro, Example 9.14]. We call it the asymptotic capacity dimension. Like the case of the capacity dimension, the following two definitions, colored and covering ones, are equivalent.

(i) The asymptotic capacity dimension $\operatorname{Cdim}(X)$ of a metric space $X$ is the minimal integer $m \geq 0$ with the following property: there is a constant $\delta \in(0,1)$ such that for every sufficiently large $R>1$ there exists an $(m+1)$-colored open covering $\mathcal{U}$ of $X$ with $\operatorname{mesh}(\mathcal{U}) \leq R$ and $L(\mathcal{U}) \geq \delta R$.

(ii) The asymptotic capacity dimension $\operatorname{Cdim}(X)$ of a metric space $X$ is the minimal integer $m \geq 0$ with the following property: there is a constant $\delta \in(0,1)$ such that for every sufficiently large $R>1$ there exists an open covering $\mathcal{U}$ of $X$ with multiplicity $m(\mathcal{U}) \leq m+1$ and such that $\operatorname{mesh}(\mathcal{U}) \leq R$ and $L(\mathcal{U}) \geq \delta R$. 
Clearly, $\operatorname{asdim} X \leq \mathrm{Cdim} X$ for any metric space $X$.

\section{§3. Auxiliary facts}

Here, we collect some facts needed for the proof of our main results.

The following lemma implies, in particular, the finite union theorem for the capacity dimension, which is similar to appropriate theorems for the asymptotic dimension [BD] and the Assouad-Nagata dimension [LS].

Lemma 3.1. Suppose that $Z$ is a metric space and $A, B \subset Z$. Let $\mathcal{U}$ be an open covering of $A$, and let $\mathcal{V}$ be an open covering of $B$, both of multiplicity at most $m$. If $\operatorname{mesh}(\mathcal{V}) \leq L(\mathcal{U}) / 2$, then there exists an open covering $\mathcal{W}$ of $A \cup B$ with multiplicity at most $m$ and $\operatorname{mesh}(\mathcal{W}) \leq \max \{\operatorname{mesh}(\mathcal{V}), \operatorname{mesh}(\mathcal{U})\}, L(\mathcal{W}) \geq \min \{L(\mathcal{U}) / 2, L(\mathcal{V})\}$.

Proof. We may assume that $L(\mathcal{U})<\infty$, i.e., no member of $\mathcal{U}$ covers $Z$, because otherwise we take $\mathcal{W}=\mathcal{U}$.

We put $r=L(\mathcal{U}) / 2$ and consider the family $\widetilde{\mathcal{U}}=\left\{B_{-r}(U): U \in \mathcal{U}\right\}$. This family still covers $A$. Next, let $\widetilde{\mathcal{V}}$ be the family of all sets $V \in \mathcal{V}$ each of which intersects some $\widetilde{U} \in \widetilde{\mathcal{U}}$. For every $\widetilde{V} \in \widetilde{\mathcal{V}}$, we fix $\widetilde{U} \in \widetilde{\mathcal{U}}$ with $\widetilde{U} \cap \widetilde{V} \neq \varnothing$ and consider the union $W=W(\widetilde{U})$ of $\widetilde{U}$ and all $\widetilde{V} \in \widetilde{\mathcal{V}}$ assigned in this way to $\widetilde{U}$. Now, we take the family $\mathcal{W}$ consisting of all $V \in \mathcal{V}$ that do not belong to $\widetilde{\mathcal{V}}$, and all $W(\widetilde{U}), \widetilde{U} \in \widetilde{\mathcal{U}}$.

By the remark at the beginning, the family $\mathcal{W}$ covers $A \cup B$. Since diam $V \leq r$ for every $V \in \mathcal{V}$, we have $W(\widetilde{U}) \subset U$ for the corresponding $U \in \mathcal{U}$, whence $\operatorname{diam} W(\widetilde{U}) \leq \operatorname{mesh} \mathcal{U}$. Hence, $\operatorname{mesh} \mathcal{W} \leq \max \{\operatorname{mesh}(\mathcal{U}), \operatorname{mesh} \mathcal{V}\}$.

Clearly, for the Lebesgue number of $\mathcal{W}$ we have

$$
L(\mathcal{W}) \geq \min \{L(\mathcal{V}), L(\widetilde{\mathcal{U}})\} \geq \min \{L(\mathcal{V}), L(\mathcal{U}) / 2\}
$$

Finally, let $\mathcal{A}$ be a collection of members of $\mathcal{W}$ with nonempty intersection. If $\mathcal{A}$ contains no member of $\mathcal{V} \backslash \widetilde{\mathcal{V}}$, then every member of $\mathcal{A}$ is contained in some member of $\mathcal{U}$, whence $|\mathcal{A}| \leq m$. Otherwise, the intersection of $\mathcal{A}$ is contained in some $V \in \mathcal{V} \backslash \widetilde{\mathcal{V}}$ and, therefore, misses the closure of any $\widetilde{U} \in \widetilde{\mathcal{U}}$. Thus, the number of members of $\mathcal{A}$ does not exceed the number of members of $\mathcal{V}$ that contain the intersection. This shows that the multiplicity of $\mathcal{W}$ is at most $m$.

We say that a metric space $Z$ is doubling at small scales if there is a constant $N \in \mathbb{N}$ such that for every sufficiently small $r>0$ every ball in $Z$ of radius $2 r$ can be covered by at most $N$ balls of radius $r$.

Similarly, a metric space $X$ is asymptotically doubling if there is a constant $N \in \mathbb{N}$ such that for every sufficiently large $R>1$, every ball in $X$ of radius $2 R$ can be covered by at most $N$ balls of radius $R$.

Lemma 3.2. (1) Assume that a metric space $Z$ is locally similar to a compact metric space $Y$. Then $Z$ is doubling at small scales.

(2) Assume that a metric space $X$ is asymptotically similar to a compact metric space $Y$. Then $X$ is asymptotically doubling.

Proof. (1) There is $\lambda \geq 1$ such that for every sufficiently large $R>1$ and every $A \subset Z$ with $\operatorname{diam} A \leq \Lambda_{0} / R, \Lambda_{0}=\min \{1, \operatorname{diam} Z / \lambda\}$, there is a $\lambda$-quasihomothetic map $f$ : $A \rightarrow Y$ with coefficient $R$.

We fix a positive $\rho \leq \Lambda_{0} /(4 \lambda)$. Since $Y$ is compact, there is $N \in \mathbb{N}$ such that any subset $B \subset Y$ can be covered by at most $N$ balls of radius $\rho$ centered at points of $B$. We take $r>0$ so small that $R=\lambda \rho / r$ satisfies the assumption above. Then for any ball $B_{2 r} \subset Z$ we have

$$
\operatorname{diam} B_{2 r} \leq 4 r \leq \Lambda_{0} / R
$$


and thus there is a $\lambda$-quasihomothetic map $f: B_{2 r} \rightarrow Y$ with coefficient $R$. The image $f\left(B_{2 r}\right)$ is covered by at most $N$ balls of radius $\rho$ centered at points of $f\left(B_{2 r}\right)$. The preimage under $f$ of every such ball is contained in a ball of radius not exceeding $\lambda \rho / R=r$ centered at a point in $B_{2 r}$. Hence, $B_{2 r}$ is covered by at most $N$ balls of radius $r$, and $Z$ is doubling at small scales.

(2) Again, there are $\Lambda_{0}, \lambda \geq 1$ such that for every sufficiently large $R>1$ and every $A \subset X$ with $\operatorname{diam} A \leq R / \Lambda_{0}$ there is a $\lambda$-quasihomothetic map $f: Y \rightarrow X$ with coefficient $R$ whose image contains an isometric copy $A^{\prime} \subset X$ of $A, A^{\prime} \subset f(Y)$.

We fix a positive $\rho \leq 1 /\left(4 \lambda \Lambda_{0}\right)$. Since $Y$ is compact, there is $N \in \mathbb{N}$ such that any subset $B \subset Y$ can be covered by at most $N$ balls of radius $\rho$ centered at points of $B$. Take $R>1$ sufficiently large so as to ensure the assumption above. Then for any ball $B_{2 R} \subset X$ of radius $2 R$, there is a $\lambda$-quasihomothetic map $f: Y \rightarrow X$ with coefficient $4 \Lambda_{0} R$ such that $f(Y)$ contains an isometric copy $B_{2 R}^{\prime}$ of $B_{2 R}$. Without loss of generality, we can assume that $B_{2 R}^{\prime}=B_{2 R}$.

Then, the preimage $B=f^{-1}\left(B_{2 R}\right)$ is covered by at most $N$ balls of radius $\rho$ centered at points of $B$. The image under $f$ of every such ball is contained in a ball of radius not exceeding $4 \lambda \Lambda_{0} \rho R \leq R$ centered at a point in $B_{2 R}$. Hence, $B_{2 R}$ is covered by at most $N$ balls of radius $R$, and $X$ is asymptotically doubling.

The idea of the following lemma is borrowed from [LS, Lemma 2.3] as well as its proof, which we give for the reader's convenience.

Lemma 3.3. Assume that a metric space $Z$ is doubling at small scales, and that a metric space $X$ is asymptotically doubling. Then $\operatorname{cdim} Z<\infty$ and $\operatorname{Cdim} X<\infty$.

Proof. By assumption, there is $n \in \mathbb{N}$ such that every ball $B_{4 r} \subset Z$ of radius $4 r$ is covered by at most $n+1$ balls $B_{r / 2}$ for all sufficiently small $r>0$. We fix a maximal $r$-separated set $Z^{\prime} \subset Z$, i.e., $\left|z z^{\prime}\right|>r$ for each distinct $z, z^{\prime} \in Z^{\prime}$. Then the family $\mathcal{U}^{\prime}=\left\{B_{r}(z): z \in Z^{\prime}\right\}$ is an open covering of $Z$.

Since every ball $B_{r / 2}$ contains at most one point of $Z^{\prime}$ and $B_{4 r}(z)$ is covered by at most $n+1$ balls $B_{r / 2}$, the ball $B_{4 r}(z)$ contains at most $n+1$ points of $Z^{\prime}$ for every $z \in Z^{\prime}$. Thus, there is a coloring $\chi: Z^{\prime} \rightarrow A,|A|=n+1$, such that $\chi(z) \neq \chi\left(z^{\prime}\right)$ for each distinct $z, z^{\prime} \in Z$ with $\left|z z^{\prime}\right|<4 r$.

For $a \in A$, we let $Z_{a}^{\prime}=\chi^{-1}(a)$ be the set of the color $a$. Then $\left|z z^{\prime}\right| \geq 4 r$ for distinct $z, z^{\prime} \in Z_{a}^{\prime}$. Putting $\mathcal{U}_{a}=\left\{B_{2 r}(z): z \in Z_{a}^{\prime}\right\}$, we obtain an open $(n+1)$-colored covering $\mathcal{U}=\bigcup_{a \in A} \mathcal{U}_{a}$ of $Z$ with $\operatorname{mesh}(\mathcal{U}) \leq 4 r$ and $L(\mathcal{U}) \geq r$. This shows that $\operatorname{cdim} Z \leq n$.

A similar argument shows that $\operatorname{Cdim} X<\infty$. We leave the details as an exercise for the reader.

We shall use the following facts, obviously implied by the definition of a quasihomothetic map.

Lemma 3.4. Let $h: Z \rightarrow Z^{\prime}$ be a $\lambda$-quasihomothetic map with coefficient $R$. Let $V \subset Z$, let $\widetilde{\mathcal{U}}$ be an open covering of $h(V)$, and let $\mathcal{U}=h^{-1}(\widetilde{\mathcal{U}})$. Then:

(1) $R \operatorname{mesh}(\mathcal{U}) / \lambda \leq \operatorname{mesh}(\widetilde{\mathcal{U}}) \leq \lambda R \operatorname{mesh}(\mathcal{U})$;

(2) $\lambda R \cdot L(\mathcal{U}) \geq L(\widetilde{\mathcal{U}}) \geq R \cdot L(\mathcal{U}) / \lambda$, where $L(\mathcal{U})$ is the Lebesgue number of $\mathcal{U}$ as a covering of $V$.

\section{$\S 4$. Proof of Theorem 1.1}

Lemmas 3.2 and 3.3 show that $\operatorname{cdim} Z=N$ is finite. Also, we may assume that $\operatorname{dim} Y=n$ is finite. There is a constant $\delta \in(0,1)$ such that for every sufficiently small $\tau>0$ there exists an $(N+1)$-colored open covering $\mathcal{V}=\bigcup_{a \in A} \mathcal{V}^{a}$ of $Z$ with $\operatorname{mesh}(\mathcal{V}) \leq \tau$ and $L(\mathcal{V}) \geq \delta \tau$. It is convenient to take $A=\{0, \ldots, N\}$ as the set of colors. 
There is a constant $\lambda \geq 1$ such that for every sufficiently large $R>1$ and every $V \subset Z$ with $\operatorname{diam} V \leq \Lambda_{0} / R, \Lambda_{0}=\min \{1, \operatorname{diam} Y / \lambda\}$, there is a $\lambda$-quasihomothetic map $h_{V}: V \rightarrow Y$ with coefficient $R$.

Using the fact that $Y$ is compact and $\operatorname{dim} Y=n$, for every $a \in A$ we find a finite open covering $\widetilde{\mathcal{U}}_{a}$ of $Y$ with multiplicity $m\left(\widetilde{\mathcal{U}}_{a}\right) \leq n+1$ such that the following is true:

(i) $\operatorname{mesh}\left(\widetilde{\mathcal{U}}_{0}\right) \leq \frac{\delta}{2 \lambda}$;

(ii) $\operatorname{mesh}\left(\widetilde{\mathcal{U}}_{a+1}\right) \leq \frac{1}{2 \lambda^{2}} \min \left\{L\left(\widetilde{\mathcal{U}}_{a}\right), \operatorname{mesh}\left(\widetilde{\mathcal{U}}_{a}\right)\right\}$ for every $a \in A, a \leq N-1$.

Then $l:=\min \left\{L\left(\widetilde{\mathcal{U}}_{N}\right), \frac{1}{2} L\left(\widetilde{\mathcal{U}}_{N-1}\right), \ldots, \frac{1}{2^{N}} L\left(\widetilde{\mathcal{U}}_{0}\right)\right\}>0$ and mesh $\left(\widetilde{\mathcal{U}}_{a}\right) \leq \frac{\delta}{2 \lambda}$ for every $a \in A$.

For every $V \in \mathcal{V}$, consider the slightly smaller subset $V^{\prime}=B_{-\delta \tau / 2}(V)$. Then the sets $Z_{a}=\bigcup_{V \in \mathcal{V}^{a}} V^{\prime} \subset Z, a \in A$, cover $Z=\bigcup_{a \in A} Z_{a}$, because $L(\mathcal{V}) \geq \delta \tau$.

Given $V \in \mathcal{V}$, we fix a $\lambda$-quasihomothetic map $h_{V}: V \rightarrow Z$ with coefficient $R=1 / \tau$ and put $\widetilde{V}=h_{V}\left(V^{\prime}\right)$. Now, for every $a \in A$ and $V \in \mathcal{V}^{a}$ consider the family $\widetilde{\mathcal{U}}_{a, V}=$ $\left\{\widetilde{U} \in \widetilde{\mathcal{U}}_{a}: \widetilde{V} \cap \widetilde{U} \neq \emptyset\right\}$, which is obviously a covering of $\widetilde{V}$ with multiplicity not exceeding $n+1$. Then

$$
\mathcal{U}_{a, V}=\left\{h_{V}^{-1}(\widetilde{U}): \widetilde{U} \in \widetilde{\mathcal{U}}_{a, V}\right\}
$$

is an open covering of $V^{\prime}$ with multiplicity at most $n+1$.

Note that $U=h_{V}^{-1}(\widetilde{U})$ is contained in $V$ for every $\widetilde{U} \in \widetilde{\mathcal{U}}_{a, V}$, because $\operatorname{dist}\left(v^{\prime}, Z \backslash V\right)>$ $\delta \tau / 2$ for every $v^{\prime} \in V^{\prime}$ and $\operatorname{diam} U \leq \lambda \tau \operatorname{diam} \widetilde{U} \leq \delta \tau / 2$. Thus, the family $\mathcal{U}_{a, V}$ is contained in $V$. Now, the family $\mathcal{U}_{a}=\bigcup_{V \in \mathcal{V}^{a}} \mathcal{U}_{a, V}$ covers the set $Z_{a}$ of the color $a$, and this family has the following properties:

(1) for every $a \in A$, the multiplicity of $\mathcal{U}_{a}$ is at most $n+1$;

(2) $\operatorname{mesh}\left(\mathcal{U}_{a+1}\right) \leq \frac{1}{2} \min \left\{L\left(\mathcal{U}_{a}\right), \operatorname{mesh}\left(\mathcal{U}_{a}\right)\right\}$ for every $a \in A, a \leq N-1\left(L\left(\mathcal{U}_{a}\right)\right.$ means the Lebesgue number of $\mathcal{U}_{a}$ viewed as a covering of $\left.Z_{a}\right)$

(3) $\operatorname{mesh}\left(\mathcal{U}_{a}\right) \leq \lambda \tau \operatorname{mesh}\left(\widetilde{\mathcal{U}}_{a}\right)$ and $L\left(\mathcal{U}_{a}\right) \geq \tau L\left(\widetilde{\mathcal{U}}_{a}\right) / \lambda$ for every $a \in A$.

Indeed, distinct $V_{1}, V_{2} \in \mathcal{V}^{a}$ are disjoint, and thus any $U_{1} \in \mathcal{U}_{a, V_{1}}, U_{2} \in \mathcal{U}_{a, V_{2}}$ are disjoint, because $U_{1} \subset V_{1}, U_{2} \subset V_{2}$. This proves (1). Furthermore, for every $a \in A$, $a \leq N-1$ and every $U \in \mathcal{U}_{a+1}$ we have

$$
\begin{aligned}
\operatorname{diam} U & \leq \lambda \tau \operatorname{mesh}\left(\widetilde{\mathcal{U}}_{a+1}\right) \leq \frac{\tau}{2 \lambda} \min \left\{L\left(\widetilde{\mathcal{U}}_{a}\right), \operatorname{mesh}\left(\widetilde{\mathcal{U}}_{a}\right)\right\} \\
& \leq \frac{1}{2} \min \left\{L\left(\mathcal{U}_{a}\right), \operatorname{mesh}\left(\mathcal{U}_{a}\right)\right\}
\end{aligned}
$$

by Lemma 3.4, which yields (2). Finally, (3) also follows from Lemma 3.4.

Now, we put $\widehat{\mathcal{U}}_{-1}=\{Z\}, \widehat{\mathcal{U}}_{0}=\mathcal{U}_{0}$ and assume that for some $a \in A$ we have already constructed families $\widehat{\mathcal{U}}_{0}, \ldots, \widehat{\mathcal{U}}_{a}$ so that $\widehat{\mathcal{U}}_{a}$ covers $Z_{0} \cup \cdots \cup Z_{a}$ with multiplicity not exceeding $n+1$ and $\operatorname{mesh}\left(\mathcal{U}_{a}\right) \leq \frac{1}{2} L\left(\widehat{\mathcal{U}}_{a-1}\right), \operatorname{mesh}\left(\widehat{\mathcal{U}}_{a}\right) \leq \operatorname{mesh}\left(\mathcal{U}_{0}\right), L\left(\widehat{\mathcal{U}}_{a}\right) \geq \min \left\{L\left(\mathcal{U}_{a}\right), \frac{1}{2} L\left(\widehat{\mathcal{U}}_{a-1}\right)\right\}$ Then, using (2), we see that

$$
\operatorname{mesh}\left(\mathcal{U}_{a+1}\right) \leq \frac{1}{2} \min \left\{L\left(\mathcal{U}_{a}\right), \frac{1}{2} L\left(\widehat{\mathcal{U}}_{a-1}\right)\right\} \leq \frac{1}{2} L\left(\widehat{\mathcal{U}}_{a}\right)
$$

Applying Lemma 3.1 to the pair of families $\widehat{\mathcal{U}}_{a}, \mathcal{U}_{a+1}$, we obtain an open covering $\widehat{\mathcal{U}}_{a+1}$ of $Z_{0} \cup \cdots \cup Z_{a+1}$ with multiplicity not exceeding $n+1$ and with

$$
\operatorname{mesh}\left(\widehat{\mathcal{U}}_{a+1}\right) \leq \max \left\{\operatorname{mesh}\left(\widehat{\mathcal{U}}_{a}\right), \operatorname{mesh}\left(\mathcal{U}_{a+1}\right)\right\} \leq \operatorname{mesh}\left(\mathcal{U}_{0}\right)
$$

and $L\left(\widehat{\mathcal{U}}_{a+1}\right) \geq \min \left\{L\left(\mathcal{U}_{a+1}\right), \frac{1}{2} L\left(\widehat{\mathcal{U}}_{a}\right)\right\}$.

Proceeding by induction and using (3), we obtain an open covering $\mathcal{U}=\widehat{\mathcal{U}}_{N}$ of $Z$ of multiplicity not exceeding $n+1$ with $\operatorname{mesh}(\mathcal{U}) \leq \operatorname{mesh}\left(\mathcal{U}_{0}\right) \leq \delta \tau / 2$ and

$$
L(\mathcal{U}) \geq \min \left\{L\left(\mathcal{U}_{N}\right), \frac{1}{2} L\left(\mathcal{U}_{N-1}\right), \ldots, \frac{1}{2^{N}} L\left(\mathcal{U}_{0}\right)\right\} \geq(l / \lambda) \tau .
$$


Since we can choose $\tau>0$ arbitrarily small and the constants $\delta, \lambda, l$ are independent of $\tau$, this shows that $\operatorname{cdim} Z \leq n$.

Proof of Corollary 1.2. We have $\operatorname{dim} Z \leq \operatorname{cdim} Z$ for every metric space $Z$. By Theorem 1.1, $\operatorname{cdim} Z<\infty$ and $\operatorname{cdim} Z \leq \operatorname{dim} Z$, whence $\operatorname{cdim} Z=\operatorname{dim} Z$ is finite.

4.1. The capacity dimension versus the topological dimension. The following proposition allows us to construct various examples of compact metric spaces for which the capacity dimension is arbitrarily larger than the topological dimension.

Proposition 4.1. Let $X, Y$ be bounded metric spaces such that for every $\varepsilon>0$ there is $A \subset X$ and a homothety $h_{\varepsilon}: A \rightarrow Y$ with $\varepsilon$-dense image, $\operatorname{dist}\left(y, h_{\varepsilon}(A)\right)<\varepsilon$ for every $y \in Y$. Then $\operatorname{cdim} X \geq \operatorname{dim} Y$.

Proof. We may assume that $\operatorname{dim} Y>0$, in particular, $\operatorname{diam} Y>0$. Then for the coefficient $\lambda(\varepsilon)$ of the homothety $h_{\varepsilon}$ we have $\lambda(\varepsilon) \geq \lambda_{0}>0$ as $\varepsilon \rightarrow 0$, because $X$ is bounded.

Assume that $n=\operatorname{cdim} X<\operatorname{dim} Y$. There is $\delta>0$ such that for every sufficiently small $\tau>0$ there is an open covering $\mathcal{U}_{\tau}$ of $X$ of multiplicity not exceeding $n+1$ with $\operatorname{mesh}\left(\mathcal{U}_{\tau}\right) \leq \tau$ and $L\left(\mathcal{U}_{\tau}\right) \geq \delta \tau$.

Using the estimate $\lambda(\varepsilon) \geq \lambda_{0}$, we can find $\tau=\tau(\varepsilon)$ such that $\lambda(\varepsilon) \tau(\varepsilon) \rightarrow 0$ as $\varepsilon \rightarrow 0$ and $\delta \lambda(\varepsilon) \tau(\varepsilon) \geq 4 \varepsilon$. Then for the covering $\mathcal{V}_{\varepsilon}=h_{\varepsilon}\left(\mathcal{U}_{\tau}\right)$ of $h_{\varepsilon}(A)$ we have $\operatorname{mesh}\left(\mathcal{V}_{\varepsilon}\right) \leq \lambda(\varepsilon) \tau(\varepsilon)$ and $L\left(\mathcal{V}_{\varepsilon}\right) \geq \delta \lambda(\varepsilon) \tau(\varepsilon)$. Furthermore, $m\left(\mathcal{V}_{\varepsilon}\right) \leq n+1$. Therefore, the family $\mathcal{V}_{\varepsilon}^{\prime}=B_{-2 \varepsilon}\left(\mathcal{V}_{\varepsilon}\right)$ still covers $h_{\varepsilon}(A)$. Taking the $\varepsilon$-neighborhood in $Y$ of every $V \in \mathcal{V}_{\varepsilon}^{\prime}$, we obtain an open covering $\mathcal{V}$ of $Y$ with $\operatorname{mesh}(\mathcal{V}) \leq \operatorname{mesh}\left(\mathcal{V}_{\varepsilon}\right) \rightarrow 0$ as $\varepsilon \rightarrow 0$.

We estimate the multiplicity of $\mathcal{V}$. Assume that $y \in Y$ is a common point of the members $V_{j} \in \mathcal{V}, j \in J$. By the definition of $\mathcal{V}$, for every $j \in J$ there is $a_{j} \in A$ such that $f\left(a_{j}\right) \in V_{j}^{\prime} \in \mathcal{V}_{\varepsilon}^{\prime}$ and $\left|f\left(a_{j}\right) y\right|<\varepsilon$. Then the mutual distances of the points $f\left(a_{j}\right), j \in J$, are less than $2 \varepsilon$. Since $V_{j}^{\prime}=B_{-2 \varepsilon}\left(U_{j}\right)$ for $U_{j} \in \mathcal{V}_{\varepsilon}$, we see that every point $f\left(a_{j}\right), j \in J$, is contained in every $U_{i}, i \in J$, which implies that $|J| \leq n+1$, because the multiplicity of $\mathcal{V}_{\varepsilon}$ is at most $n+1$. Hence, $m(\mathcal{V}) \leq n+1$ and $\operatorname{dim} Y \leq n$, a contradiction.

As an application, we obtain the following examples. Let $Z=\{0\} \cup\{1 / m: m \in \mathbb{N}\}$. Then $\operatorname{cdim} Z^{n}=n$ for any $n \geq 1$, while $\operatorname{dim} Z^{n}=0$. Indeed, obviously, the spaces $X=Z^{n}$ and $Y=[0,1]^{n}$ satisfy the condition of Proposition 4.1, whence $\operatorname{cdim} Z^{n} \geq$ $\operatorname{dim} Y=n$ (we have equality here because $Z^{n} \subset Y$ ). For $n=1$, this example was given in $[\mathrm{LS}]$ in the context of the Assouad-Nagata dimension.

Using Corollary 1.2 and the quasisymmetry invariance of the capacity dimension (see [Bu1]), we conclude that the space $Z^{n}$ is not quasisymmetric to any locally self-similar space for any $n \geq 1$.

Further examples. We take any monotone sequence of positive $\varepsilon_{k} \rightarrow 0, \varepsilon_{1}=1 / 3$, and repeat the construction of the standard ternary Cantor set $K \subset[0,1]$, but, instead of the $(1 / 3)^{k}$-length intervals, at every $k$ th step, $k \geq 1$, we remove the middle intervals of length $s_{k}=\varepsilon_{k} l_{k}, l_{1}=1$, where the length $l_{k+1}$ of the segments obtained after processing the $k$ th step is defined recursively by $2 l_{k+1}+s_{k}=l_{k}$. The resulting compact space $K_{a} \subset[0,1]$ is homeomorphic to $K$. It is easily seen that cdim $K=0$. However, $X=K_{a}$ and $Y=[0,1]$ satisfy the condition of Proposition 4.1, so that $\operatorname{cdim} K_{a}=1$, while $\operatorname{dim} K_{a}=0$.

Similarly, one can construct "exotic" Sierpinski carpets, Menger curves, etc. with capacity dimension strictly greater than the topological dimension. Each of those compact metric spaces is not quasisymmetric to any locally self-similar space; in particular, it is not quasisymmetric to the boundary at infinity (with a visual metric) of a hyperbolic group; see Theorem 1.4. For comparison, it is well known that the boundary at infinity of a typical hyperbolic group is homeomorphic to the Menger curve. 


\section{§5. Proof of Theorem 1.3}

In fact, we prove that under the condition of Theorem 1.3, the following three dimensions are finite and coincide:

$$
\operatorname{asdim} X=\mathrm{Cdim} X=\operatorname{dim} Y .
$$

We have $\operatorname{asdim} X \leq \operatorname{Cdim} X$ for every metric space $X$, and $\operatorname{Cdim} X$ is finite by Lemmas 3.2 and 3.3, because $X$ is asymptotically similar to the compact space $Y$. First, we show that $\operatorname{dim} Y \leq \operatorname{asdim} X$. Let $\operatorname{asdim} X=N$. Then for every sufficiently large $\tau>1$ there exists an open covering $\mathcal{V}$ of $X$ with multiplicity not exceeding $N+1$ and $\operatorname{such}$ that $\operatorname{mesh}(\mathcal{V})<\infty$ and $L(\mathcal{V}) \geq \tau$.

There are constants $\Lambda_{0}, \lambda \geq 1$ such that for every sufficiently large $R>1$ and every $V \subset X$ with $\operatorname{diam} V \leq R / \Lambda_{0}$, there is a $\lambda$-quasihomothetic map $h_{V}: Y \rightarrow X$ with coefficient $R$ whose image contains an isometric copy $V^{\prime} \subset X$ of $V, V^{\prime} \subset h_{V}(Y)$.

Given $\varepsilon>0$, we take a sufficiently large $R>1$ satisfying the condition above and such that $\frac{\lambda}{R} \operatorname{mesh}(\mathcal{V})<\varepsilon$. Then there is a $\lambda$-quasihomothetic map $h: Y \rightarrow X$ with coefficient $R$. The family $\mathcal{U}=h^{-1}(\mathcal{V})$ is an open covering of $Y$ with multiplicity not exceeding $N+1$ and with $\operatorname{mesh}(\mathcal{U}) \leq \frac{\lambda}{R} \operatorname{mesh}(\mathcal{V})<\varepsilon$. Consequently, $\operatorname{dim} Y \leq \operatorname{asdim} X$.

It remains to show that $\operatorname{Cdim} X \leq \operatorname{dim} Y$. We already know that the topological dimension of $Y$ and the asymptotic capacity dimension of $X$ are finite; we put $\operatorname{dim} Y=n$, $\operatorname{Cdim} X=N$. Starting with this point, the proof is completely parallel to that of Theorem 1.1.

In accordance with the definition of Cdim, there is $\delta \in(0,1)$ such that for every sufficiently large $R$ there exists an $(N+1)$-colored open covering $\mathcal{V}=\bigcup_{a \in A} \mathcal{V}^{a}$ of $X$ with $\operatorname{mesh}(\mathcal{V}) \leq R$ and $L(\mathcal{V}) \geq \delta R$. It is convenient to take $A=\{0, \ldots, N\}$ as the set of colors.

Since $Y$ is compact and $\operatorname{dim} Y=n$, for every $a \in A$ we can find a finite open covering $\widetilde{\mathcal{U}}_{a}$ of $Y$ having multiplicity $m\left(\widetilde{\mathcal{U}}_{a}\right) \leq n+1$ and such that the following is true:

(i) $\operatorname{mesh}\left(\widetilde{\mathcal{U}}_{0}\right) \leq \frac{\delta}{2 \lambda \Lambda_{0}}$;

(ii) $\operatorname{mesh}\left(\widetilde{\mathcal{U}}_{a+1}\right) \leq \frac{1}{2 \lambda^{2}} \min \left\{L\left(\widetilde{\mathcal{U}}_{a}\right), \operatorname{mesh}\left(\widetilde{\mathcal{U}}_{a}\right)\right\}$ for every $a \in A, a \leq N-1$.

Then $l:=\min \left\{L\left(\widetilde{\mathcal{U}}_{N}\right), \frac{1}{2} L\left(\widetilde{\mathcal{U}}_{N-1}\right), \ldots, \frac{1}{2^{N}} L\left(\widetilde{\mathcal{U}}_{0}\right)\right\}>0$ and $\operatorname{mesh}\left(\widetilde{\mathcal{U}}_{a}\right) \leq \frac{\delta}{2 \lambda \Lambda_{0}}$ for every $a \in A$.

We put $t=\delta R / 2$ and for every $V \in \mathcal{V}$ consider the smaller subset $V_{t}=B_{-t}(V)$. Then the sets $X_{a}=\bigcup_{V \in \mathcal{V}^{a}} V_{t} \subset X, a \in A$, cover $X=\bigcup_{a \in A} X_{a}$, because $L(\mathcal{V}) \geq \delta R=2 t$.

For any $V \in \mathcal{V}$ there is a $\lambda$-quasihomothetic map $h_{V}: Y \rightarrow X$ with coefficient $\Lambda_{0} R$ such that $V^{\prime} \subset h_{V}(Y)$ for some isometric copy $V^{\prime} \subset X$ of $V$. Taking the inverse, we obtain a $\lambda$-quasihomothetic map $f_{V}: V \rightarrow Y$ with coefficient $\left(\Lambda_{0} R\right)^{-1}$.

Now, for every $a \in A$ and $V \in \mathcal{V}^{a}$, consider the family

$$
\mathcal{U}_{a, V}=\left\{f_{V}^{-1}(\widetilde{U}): \widetilde{U} \in \widetilde{\mathcal{U}}_{a}, f_{V}\left(V_{t}\right) \cap \widetilde{U} \neq \varnothing\right\},
$$

which is obviously a covering of $V_{t}$ with multiplicity at most $n+1$.

Observe that every $U \in \mathcal{U}_{a, V}, U=f_{V}^{-1}(\widetilde{U})$, is contained in $V$, because $\operatorname{dist}(v, X \backslash V)>$ $t$ for every $v \in V_{t}$ and $\operatorname{diam} U \leq \lambda \Lambda_{0} R \operatorname{diam} \widetilde{U} \leq t$. Thus, the family $\mathcal{U}_{a, V}$ is contained in $V$. Now, the family $\mathcal{U}_{a}=\bigcup_{V \in \mathcal{V}^{a}} \mathcal{U}_{a, V}$ covers the set $X_{a}$ of the color $a$, and it has the following properties:

(1) for every $a \in A$, the multiplicity of $\mathcal{U}_{a}$ is at most $n+1$;

(2) $\operatorname{mesh}\left(\mathcal{U}_{a+1}\right) \leq \frac{1}{2} \min \left\{L\left(\mathcal{U}_{a}\right), \operatorname{mesh}\left(\mathcal{U}_{a}\right)\right\}$ for every $a \in A, a \leq N-1\left(L\left(\mathcal{U}_{a}\right)\right.$ means the Lebesgue number of $\mathcal{U}_{a}$ viewed as a covering of $\left.X_{a}\right)$;

(3) $\operatorname{mesh}\left(\mathcal{U}_{a}\right) \leq \lambda \Lambda_{0} R \operatorname{mesh}\left(\widetilde{\mathcal{U}}_{a}\right)$ and $L\left(\mathcal{U}_{a}\right) \geq \Lambda_{0} R \cdot L\left(\widetilde{\mathcal{U}}_{a}\right) / \lambda$ for every $a \in A$. 
For (1) and (3), the argument is literally the same as in the proof of Theorem 1.1. Next, for every $a \in A, a \leq N-1$, and every $U \in \mathcal{U}_{a+1}$ we have

$$
\begin{aligned}
\operatorname{diam} U & \leq \lambda \Lambda_{0} R \operatorname{mesh}\left(\widetilde{\mathcal{U}}_{a+1}\right) \leq \frac{\Lambda_{0} R}{2 \lambda} \min \left\{L\left(\widetilde{\mathcal{U}}_{a}\right), \operatorname{mesh}\left(\widetilde{\mathcal{U}}_{a}\right)\right\} \\
& \leq \frac{1}{2} \min \left\{L\left(\mathcal{U}_{a}\right), \operatorname{mesh}\left(\mathcal{U}_{a}\right)\right\}
\end{aligned}
$$

by Lemma 3.4, and (2) follows.

Now, we put $\widehat{\mathcal{U}}_{-1}=\{X\}$ and $\widehat{\mathcal{U}}_{0}=\mathcal{U}_{0}$, and assume that for some $a \in A$ we have already constructed families $\widehat{\mathcal{U}}_{0}, \ldots, \widehat{\mathcal{U}}_{a}$ so that $\widehat{\mathcal{U}}_{a}$ covers $X_{0} \cup \cdots \cup X_{a}$ with multiplicity not exceeding $n+1$ and with $\operatorname{mesh}\left(\mathcal{U}_{a}\right) \leq \frac{1}{2} L\left(\widehat{\mathcal{U}}_{a-1}\right), \operatorname{mesh}\left(\widehat{\mathcal{U}}_{a}\right) \leq \operatorname{mesh}\left(\mathcal{U}_{0}\right)$, and $L\left(\widehat{\mathcal{U}}_{a}\right) \geq$ $\min \left\{L\left(\mathcal{U}_{a}\right), \frac{1}{2} L\left(\widehat{\mathcal{U}}_{a-1}\right)\right\}$. Then, using $(2)$, we obtain

$$
\operatorname{mesh}\left(\mathcal{U}_{a+1}\right) \leq \frac{1}{2} \min \left\{L\left(\mathcal{U}_{a}\right), \frac{1}{2} L\left(\widehat{\mathcal{U}}_{a-1}\right)\right\} \leq \frac{1}{2} L\left(\widehat{\mathcal{U}}_{a}\right)
$$

Applying Lemma 3.1 to the pair of families $\widehat{\mathcal{U}}_{a}, \mathcal{U}_{a+1}$, we obtain an open covering $\widehat{\mathcal{U}}_{a+1}$ of $X_{0} \cup \cdots \cup X_{a+1}$ with multiplicity at most $n+1$ and with $\operatorname{mesh}\left(\widehat{\mathcal{U}}_{a+1}\right) \leq$ $\max \left\{\operatorname{mesh}\left(\widehat{\mathcal{U}}_{a}\right), \operatorname{mesh}\left(\mathcal{U}_{a+1}\right)\right\} \leq \operatorname{mesh}\left(\mathcal{U}_{0}\right)$ and $L\left(\widehat{\mathcal{U}}_{a+1}\right) \geq \min \left\{L\left(\mathcal{U}_{a+1}\right), \frac{1}{2} L\left(\widehat{\mathcal{U}}_{a}\right)\right\}$.

Proceeding by induction and using (3), we obtain an open covering $\mathcal{U}=\widehat{\mathcal{U}}_{N}$ of $X$ of multiplicity at most $n+1$ with $\operatorname{mesh}(\mathcal{U}) \leq \operatorname{mesh}\left(\mathcal{U}_{0}\right) \leq \delta R / 2$ and $L(\mathcal{U}) \geq$ $\min \left\{L\left(\mathcal{U}_{N}\right), \frac{1}{2} L\left(\mathcal{U}_{N-1}\right), \ldots, \frac{1}{2^{N}} L\left(\mathcal{U}_{0}\right)\right\} \geq\left(l \Lambda_{0} / \lambda\right) R$. Since we can choose $R$ arbitrarily large and the constants $\delta, \lambda, \Lambda_{0}, l$ are independent of $R$, this shows that $\operatorname{Cdim} X \leq n$.

\section{$\S 6$. Applications}

\subsection{Capacity dimension of the boundary at infinity of a hyperbolic group.}

Here, we describe a large class of hyperbolic spaces whose boundary at infinity is locally self-similar and prove a generalization of Theorem 1.4.

We recall some necessary facts from the theory of hyperbolic spaces. For more details the reader may consult, e.g., [BoS]. We assume that the reader is familiar with notions such as a geodesic metric space, a triangle, a geodesic ray, etc.

Let $X$ be a geodesic metric space. We write $x x^{\prime}$ for a geodesic in $X$ between $x$, $x^{\prime} \in X$, and $\left|x x^{\prime}\right|$ for the distance between them. For $o \in X$ and $x, x^{\prime} \in X$, we put $\left(x \mid x^{\prime}\right)_{o}=\frac{1}{2}\left(|x o|+\left|x^{\prime} o\right|-\left|x x^{\prime}\right|\right)$. The number $\left(x \mid x^{\prime}\right)_{o}$ is nonnegative by the triangle inequality; it is called the Gromov product of $x, x^{\prime}$ with respect to $o$.

Lemma 6.1. Let $o, g, x^{\prime}, x^{\prime \prime}$ be points of a metric space $X$, and let $\left(x^{\prime} \mid g\right)_{o},\left(x^{\prime \prime} \mid g\right)_{o} \geq$ $|o g|-\sigma$ for some $\sigma \geq 0$. Then

$$
\left(x^{\prime} \mid x^{\prime \prime}\right)_{o} \leq\left(x^{\prime} \mid x^{\prime \prime}\right)_{g}+|o g| \leq\left(x^{\prime} \mid x^{\prime \prime}\right)_{o}+2 \sigma .
$$

Proof. The left-hand inequality follows immediately from the triangle inequality: since $\left|o x^{\prime}\right| \leq|o g|+\left|g x^{\prime}\right|$ and $\left|o x^{\prime \prime}\right| \leq|o g|+\left|g x^{\prime \prime}\right|$, we have $\left(x^{\prime} \mid x^{\prime \prime}\right)_{o} \leq\left(x^{\prime} \mid x^{\prime \prime}\right)_{g}+|o g|$.

Next, we note that $\left(x^{\prime} \mid o\right)_{g}=|o g|-\left(x^{\prime} \mid g\right)_{o} \leq \sigma$. This yields $\left|x^{\prime} o\right|=|o g|+\left|g x^{\prime}\right|-$ $2\left(x^{\prime} \mid o\right)_{g} \geq|o g|+\left|g x^{\prime}\right|-2 \sigma$ and similarly $\left|x^{\prime \prime} o\right| \geq|o g|+\left|g x^{\prime \prime}\right|-2 \sigma$. Now, the right-hand inequality follows.

A geodesic metric space $X$ is said to be $\delta$-hyperbolic, $\delta \geq 0$, if the following is true for any triangle $x y z \subset X$ : if $y^{\prime} \in x y, z^{\prime} \in x z$ satisfy $\left|x y^{\prime}\right|=\left|x z^{\prime}\right| \leq(y \mid z)_{x}$, then $\left|y^{\prime} z^{\prime}\right| \leq \delta$. In this case, the $\delta$-inequality

$$
(x \mid y)_{o} \geq \min \left\{(x \mid z)_{o},(z \mid y)_{o}\right\}-\delta
$$

is fulfilled for every base point $o \in X$ and all $x, y, z \in X$. A geodesic space is (Gromov) hyperbolic if it is $\delta$-hyperbolic for some $\delta \geq 0$. 
Let $X$ be a $\delta$-hyperbolic space and $o \in X$ a base point. A sequence of points $\left\{x_{i}\right\} \subset X$ converges to infinity if

$$
\lim _{i, j \rightarrow \infty}\left(x_{i} \mid x_{j}\right)_{o}=\infty .
$$

Two sequences $\left\{x_{i}\right\},\left\{x_{i}^{\prime}\right\}$ that converge to infinity are equivalent if

$$
\lim _{i \rightarrow \infty}\left(x_{i} \mid x_{i}^{\prime}\right)_{o}=\infty
$$

By using the $\delta$-inequality, it is easy to show that this defines an equivalence relation for sequences in $X$ converging to infinity. The boundary at infinity $\partial_{\infty} X$ of $X$ is defined as the set of equivalence classes of sequences converging to infinity. Every isometry of $X$ extends canonically to a bijection of $\partial_{\infty} X$ onto itself, and we use the same notation for the extension.

Every geodesic ray in $X$ represents a point at infinity. Conversely, if a geodesic hyperbolic space $X$ is proper (i.e., the closed balls in $X$ are compact), then every point at infinity is represented by a geodesic ray.

The Gromov product extends to $X \cup \partial_{\infty} X$ as follows. For points $\xi, \xi^{\prime} \in \partial_{\infty} X$, we define

$$
\left(\xi \mid \xi^{\prime}\right)_{o}=\inf \liminf _{i \rightarrow \infty}\left(x_{i} \mid x_{i}^{\prime}\right)_{o}
$$

where the infimum is taken over all sequences $\left\{x_{i}\right\} \in \xi,\left\{x_{i}^{\prime}\right\} \in \xi^{\prime}$. Note that $\left(\xi \mid \xi^{\prime}\right)_{o}$ takes values in $[0, \infty]$, and that $\left(\xi \mid \xi^{\prime}\right)_{o}=\infty$ if and only if $\xi=\xi^{\prime}$.

Similarly, the Gromov product

$$
(x \mid \xi)_{o}=\inf \liminf _{i \rightarrow \infty}\left(x \mid x_{i}\right)_{o}
$$

is defined for any $x \in X, \xi \in \partial_{\infty} X$, where the infimum is taken over all sequences $\left\{x_{i}\right\} \in \xi$.

Furthermore, for any $\xi, \xi^{\prime} \in X \cup \partial_{\infty} X$ and for arbitrary sequences $\left\{x_{i}\right\} \in \xi,\left\{x_{i}^{\prime}\right\} \in \xi^{\prime}$, we have

$$
\left(\xi \mid \xi^{\prime}\right)_{o} \leq \liminf _{i \rightarrow \infty}\left(x_{i} \mid x_{i}^{\prime}\right)_{o} \leq \limsup _{i \rightarrow \infty}\left(x_{i} \mid x_{i}^{\prime}\right)_{o} \leq\left(\xi \mid \xi^{\prime}\right)_{o}+2 \delta .
$$

Moreover, the $\delta$-inequality is fulfilled in $X \cup \partial_{\infty} X$ :

$$
\left(\xi \mid \xi^{\prime \prime}\right)_{o} \geq \min \left\{\left(\xi \mid \xi^{\prime}\right)_{o},\left(\xi^{\prime} \mid \xi^{\prime \prime}\right)_{o}\right\}-\delta
$$

for every $\xi, \xi^{\prime}, \xi^{\prime \prime} \in X \cup \partial_{\infty} X$.

A metric $d$ on the boundary at infinity $\partial_{\infty} X$ of $X$ is said to be visual if there are $o \in X, a>1$, and positive constants $c_{1}, c_{2}$ such that

$$
c_{1} a^{-\left(\xi \mid \xi^{\prime}\right)_{o}} \leq d\left(\xi, \xi^{\prime}\right) \leq c_{2} a^{-\left(\xi \mid \xi^{\prime}\right)_{o}}
$$

for all $\xi, \xi^{\prime} \in \partial_{\infty} X$. In this case, we say that $d$ is the visual metric with respect to the base point $o$ and the parameter $a$. The boundary at infinity is bounded and complete with respect to any visual metric, and moreover, if $X$ is proper, then $\partial_{\infty} X$ is compact. If $a>1$ is sufficiently close to 1 , then a visual metric with respect to $a$ does exist.

A metric space $X$ is cobounded if there is a bounded subset $A \subset X$ such that the orbit of $A$ under the isometry group of $X$ covers $X$.

Proposition 6.2. The boundary at infinity of every cobounded, hyperbolic, proper, geodesic space $X$ is locally self-similar with respect to any visual metric.

Proof. We may assume that the geodesic space $X$ is $\delta$-hyperbolic, $\delta \geq 0$, and that a visual metric $d$ on $\partial_{\infty} X$ satisfies

$$
c^{-1} a^{-\left(\xi \mid \xi^{\prime}\right)_{o}} \leq d\left(\xi, \xi^{\prime}\right) \leq c a^{-\left(\xi \mid \xi^{\prime}\right)_{o}}
$$

for some base point $o \in X$, some constants $c \geq 1, a>1$, and all $\xi, \xi^{\prime} \in \partial_{\infty} X$. Note that then $\operatorname{diam} \partial_{\infty} X \leq c$. 
There is $\rho>0$ such that the orbit of the ball $B_{\rho}(o)$ under the isometry group of $X$ covers $X$. Now, we put $\lambda=c^{2} a^{\rho+4 \delta}$. Then

$$
\Lambda_{0}=\min \left\{1, \operatorname{diam} \partial_{\infty} X / \lambda\right\} \leq 1 / c .
$$

Fix $R>1$ and consider $A \subset \partial_{\infty} X$ with $\operatorname{diam} A \leq \Lambda_{0} / R$. For every $\xi, \xi^{\prime} \in A$ we have

$$
\left(\xi \mid \xi^{\prime}\right)_{o} \geq \log _{a} \frac{R}{c \Lambda_{0}} \geq \log _{a} R .
$$

We fix $\xi \in A$. Since $X$ is proper, there is a geodesic ray $o \xi \subset X$ representing $\xi$. We take $g \in o \xi$ with $a^{|o g|}=R$. The $\delta$-inequality shows that

$$
\left(\xi^{\prime} \mid g\right)_{o} \geq \min \left\{\left(\xi^{\prime} \mid \xi\right)_{o},(\xi \mid g)_{o}\right\}-\delta=|o g|-\delta
$$

for every $\xi^{\prime} \in A$, because $(\xi \mid g)_{o}=|o g|$.

For arbitrary $\xi^{\prime}, \xi^{\prime \prime} \in A$, consider sequences $\left\{x^{\prime}\right\} \in \xi^{\prime},\left\{x^{\prime \prime}\right\} \in \xi^{\prime \prime}$ such that $\left(x^{\prime} \mid x^{\prime \prime}\right)_{g} \rightarrow$ $\left(\xi^{\prime} \mid \xi^{\prime \prime}\right)_{g}$. There is no loss of generality in assuming that $\left(x^{\prime} \mid g\right)_{o},\left(x^{\prime \prime} \mid g\right)_{o} \geq|o g|-\delta$, because possible errors in these estimates disappear while taking the limit; see below.

Applying Lemma 6.1 to the points $o, g, x^{\prime}, x^{\prime \prime} \in X$ and $\sigma=\delta$, we obtain

$$
\left(x^{\prime} \mid x^{\prime \prime}\right)_{o}-|o g| \leq\left(x^{\prime} \mid x^{\prime \prime}\right)_{g} \leq\left(x^{\prime} \mid x^{\prime \prime}\right)_{o}-|o g|+2 \delta .
$$

Passage to the limit yields

$$
\left(\xi^{\prime} \mid \xi^{\prime \prime}\right)_{o}-|o g| \leq\left(\xi^{\prime} \mid \xi^{\prime \prime}\right)_{g} \leq\left(\xi^{\prime} \mid \xi^{\prime \prime}\right)_{o}-|o g|+4 \delta .
$$

There is an isometry $f: X \rightarrow X$ with $|o f(g)| \leq \rho$. Then

$$
\left(\xi^{\prime} \mid \xi^{\prime \prime}\right)_{g}-\rho \leq\left(f\left(\xi^{\prime}\right) \mid f\left(\xi^{\prime \prime}\right)\right)_{o} \leq\left(\xi^{\prime} \mid \xi^{\prime \prime}\right)_{g}+\rho,
$$

because the Gromov products with respect to different points differ one from another at most by the distance between the points. The last two double inequalities give

$$
\left(\xi^{\prime} \mid \xi^{\prime \prime}\right)_{o}-|o g|-\rho \leq\left(f\left(\xi^{\prime}\right) \mid f\left(\xi^{\prime \prime}\right)\right)_{o} \leq\left(\xi^{\prime} \mid \xi^{\prime \prime}\right)_{o}-|o g|+\rho+4 \delta,
$$

whence

$$
c^{-2} a^{-(\rho+4 \delta)} R d\left(\xi^{\prime}, \xi^{\prime \prime}\right) \leq d\left(f\left(\xi^{\prime}\right), f\left(\xi^{\prime \prime}\right)\right) \leq c^{2} a^{\rho} R d\left(\xi^{\prime}, \xi^{\prime \prime}\right) .
$$

This shows that $f: A \rightarrow \partial_{\infty} X$ is $\lambda$-quasi-homothetic with coefficient $R$, and hence $\partial_{\infty} X$ is locally self-similar.

Now, Corollary 1.2 and Proposition 6.2 give the following.

Theorem 6.3. The capacity dimension of the boundary at infinity of every cobounded, hyperbolic, proper, geodesic space $X$ coincides with the topological dimension of $X$, $\operatorname{cdim} \partial_{\infty} X=\operatorname{dim} \partial_{\infty} X$.

The class of spaces satisfying the condition of Theorem 6.3 is very large. In particular, it includes all symmetric rank-one spaces of noncompact type (i.e., the real, complex, quaternionic hyperbolic spaces and the Cayley hyperbolic plane), all cocompact Hadamard manifolds of negative sectional curvature, various hyperbolic buildings, etc. The most important among them is the class of (Gromov) hyperbolic groups, and Theorem 1.4 is a particular case of Theorem 6.3 .

Note that for many such spaces, the boundary at infinity is fractal in the sense that its Hausdorff dimension with respect to a natural visual metric is greater than the topological dimension. This is true, e.g., for the complex, quaternionic hyperbolic spaces, the Cayley hyperbolic plane, the Fuchsian hyperbolic buildings (see [Bou]), and the hyperbolic graph surfaces (see $[\mathrm{Bu} 3])$. 
6.2. The asymptotic dimension of a hyperbolic group. Theorem 6.3 is the decisive step in the proof of the following statement.

Theorem 6.4. The asymptotic dimension of every cobounded, hyperbolic, proper, geodesic space $X$ equals the topological dimension of its boundary at infinity plus 1 ,

$$
\operatorname{asdim} X=\operatorname{dim} \partial_{\infty} X+1
$$

Theorem 1.5 is a particular case of Theorem 6.4. The fact that $\operatorname{asdim} X$ and $\operatorname{dim} \partial_{\infty} X$ are finite is well known; it follows, e.g., from [BoS] (for hyperbolic groups, there is an alternative proof in [Ro, Theorem 9.25]). Our contribution is that we prove the optimal estimate, $\operatorname{asdim} X \leq \operatorname{dim} \partial_{\infty} X+1$.

The estimate from below,

$$
\operatorname{asdim} X \geq \operatorname{dim} \partial_{\infty} X+1
$$

or at least the idea of its proof, is also well known; see $\left[\mathrm{Gr}, 1 . \mathrm{E}_{1}^{\prime}\right]$. Stronger estimates of different types were obtained in $[\mathrm{Sw}]$ and [BS2], respectively. For the reader's convenience, we give a simpler version of the arguments in [BS2], adapted to the asymptotic dimension.

Let $Z$ be a bounded metric space. Assuming that $\operatorname{diam} Z>0$, we put $\mu=\pi / \operatorname{diam} Z$ and observe that $\mu\left|z z^{\prime}\right| \in[0, \pi]$ for every $z, z^{\prime} \in Z$. Recall that the hyperbolic cone $\operatorname{Co}(Z)$ over $Z$ is the space $Z \times[0, \infty) / Z \times\{0\}$ with the metric defined as follows. Given $x=(z, t)$, $x^{\prime}=\left(z^{\prime}, t^{\prime}\right) \in \operatorname{Co}(Z)$, we consider a triangle $\bar{o} \bar{x} \bar{x}^{\prime} \subset \mathrm{H}^{2}$ with $|\bar{o} \bar{x}|=t,\left|\bar{o} \bar{x}^{\prime}\right|=t^{\prime}$, and the angle $\angle_{\bar{o}}\left(\bar{x}, \bar{x}^{\prime}\right)=\mu\left|z z^{\prime}\right|$. Now, we put $\left|x x^{\prime}\right|:=\left|\bar{x} \bar{x}^{\prime}\right|$. In the degenerate case $Z=\{\mathrm{pt}\}$, we define $\operatorname{Co}(Z)=\{\mathrm{pt}\} \times[0, \infty)$ as the metric product. The point $o=Z \times\{0\} \in \operatorname{Co}(Z)$ is called the vertex of $\operatorname{Co}(Z)$.

Proposition 6.5. For every geodesic hyperbolic space $X$ we have

$$
\operatorname{asdim} X \geq \operatorname{dim} \partial_{\infty} X+1
$$

Proof. The same argument as in [Bu1, Proposition 6.2] shows that the hyperbolic cone $\operatorname{Co}(Z)$ over $Z=\partial_{\infty} X$, taken with some visual metric, can be quasi-isometrically (actually, roughly similarly) embedded in $X$ because $X$ is geodesic. Thus, $\operatorname{asdim} X \geq$ asdim $\operatorname{Co}(Z)$, and we show that $\operatorname{asdim} \operatorname{Co}(Z) \geq \operatorname{dim} Z+1$.

The annulus $\operatorname{An}(Z) \subset \operatorname{Co}(Z)$ consists of all $x \in \operatorname{Co}(Z)$ with $1 \leq|x o| \leq 2$. Clearly, $\operatorname{An}(Z)$ is homeomorphic to $Z \times I, I=[0,1]$. By a well-known result of dimension theory (see $[\mathrm{Al}])$, for the topological dimension we have

$$
\operatorname{dim} \operatorname{An}(Z)=\operatorname{dim} Z+1 .
$$

Consider the sequence of contracting homeomorphisms $F_{k}: \mathrm{Co}(Z) \rightarrow \mathrm{Co}(Z)$ given by $F_{k}(z, t)=\left(z, \frac{1}{k} t\right),(z, t) \in \operatorname{Co}(Z), k \in \mathbb{N}$. For a uniformly bounded covering $\mathcal{U}$ of $\operatorname{Co}(Z)$, the coverings $\mathcal{U}_{k}=F_{k}(\mathcal{U}) \cap \operatorname{An}(Z)$ of the annulus $\operatorname{An}(Z)$ have arbitrarily small mesh as $k \rightarrow \infty$. Therefore, asdim $\operatorname{Co}(Z) \geq \operatorname{dim} \operatorname{An}(Z)$, and the estimate follows.

Proof of Theorem 6.4. The lower estimate

$$
\operatorname{asdim} X \geq \operatorname{dim} \partial_{\infty} X+1
$$

follows from Proposition 6.5. By the main result of [Bu1] (see also [Bu2] for another proof), we have $\operatorname{asdim} X \leq \operatorname{cdim} \partial_{\infty} X+1$ (certainly, the space $X$ is visual, i.e., $X$ satisfies the condition of the theorems cited). Now, the upper estimate

$$
\operatorname{asdim} X \leq \operatorname{dim} \partial_{\infty} X+1
$$

follows from Theorem 6.3. 
6.3. Embedding and nonembedding results. Combining Theorem 6.3 with the main result of $[\mathrm{Bu} 2]$, we arrive at the following statement.

Theorem 6.6. Every cobounded, hyperbolic, proper, geodesic space $X$ admits a quasiisometric embedding $X \rightarrow T_{1} \times \cdots \times T_{n}$ into the $n$-fold product of simplicial metric trees $T_{1}, \ldots, T_{n}$ with $n=\operatorname{dim} \partial_{\infty} X+1$.

(Every space $X$ satisfying the condition of Theorem 6.6 is certainly visual; i.e., $X$ also satisfies the condition of [Bu2, Theorem 1.1].) For example, the complex hyperbolic plane $\mathbb{C H}^{2}$ admits a quasi-isometric embedding into the 4 -fold product of simplicial metric trees, etc.

Theorem 1.6 is a particular case of Theorem 6.6 .

Theorem 6.3 has applications also to nonembedding results. For example, let $X^{n}$ be a universal covering of a compact Riemannian $n$-dimensional $(n \geq 2)$ manifold with nonempty geodesic boundary and constant sectional curvature -1 . Then $X^{n}$ satisfies the condition of Theorem 6.3 , and hence $\operatorname{dim} \partial_{\infty} X^{n}=\operatorname{cdim} \partial_{\infty} X^{n}$. Note that $X^{n}$ can be obtained from the real hyperbolic space $\mathrm{H}^{n}$ by removing a countable collection of disjoint open half-spaces, and $\partial_{\infty} X^{n} \subset S^{n-1}$ is a compact nowhere dense subset obtained by removing a countable collection of disjoint open balls. In particular, for $n=2, \partial_{\infty} X^{n} \subset S^{1}$ is a Cantor set, for $n=3, \partial_{\infty} X^{n} \subset S^{2}$ is a Sierpinski carpet, and for $n \geq 4, \partial_{\infty} X^{n} \subset S^{n-1}$ is a higher-dimensional version of a Sierpinski carpet. Thus, $\operatorname{dim} \partial_{\infty} X^{n}=n-2$.

The space $X^{n}$ contains isometrically embedded copies of $\mathrm{H}^{n-1}$ as the boundary components. Therefore, by [BF], the $k$-fold product $X^{n} \times \cdots \times X^{n}, k \geq 1$ factors, contains quasi-isometrically embedded $\mathrm{H}^{p}$ for $p=k(n-2)+1$.

Theorem 6.7. Let $X^{n}$ be the space as above, and let $Y_{k}^{n}=X^{n} \times \cdots \times X^{n}$ be the $k$-fold product, $k \geq 1$. Then there is no quasi-isometric embedding

$$
\mathrm{H}^{p} \rightarrow Y_{k}^{n} \times \mathbb{R}^{m}
$$

for $p>k(n-1)$ and any $m \geq 0$.

For example, this theorem says that there is no way to embed $\mathrm{H}^{5}$ quasi-isometrically into $X^{3} \times X^{3} \times \mathbb{R}^{m}$ for any $m \geq 0$, though there is a quasi-isometric embedding $\mathrm{H}^{3} \rightarrow X^{3} \times$ $X^{3}$. In general, for arbitrary $n \geq 2$, there is a quasi-isometric embedding $\mathrm{H}^{p} \rightarrow X^{n} \times X^{n}$ for $p=2 n-3$ and there is no quasi-isometric embedding $\mathrm{H}^{p} \rightarrow X^{n} \times X^{n} \times \mathbb{R}^{m}$ for $p=2 n-1$ and an arbitrary $m \geq 0$.

For $n=2$, the space $X^{2}$ is quasi-isometric to the binary tree $T$ all of whose edges have length 1 , because $X^{2}$ covers a compact hyperbolic surface with nonempty geodesic boundary. By [DS], there is a quasi-isometric embedding $\mathrm{H}^{2} \rightarrow T \times T$, and hence there is a quasi-isometric embedding $\mathrm{H}^{p} \rightarrow X^{n} \times X^{n}$ in the remaining case $p=2 n-2$ if $n=2$. For $n \geq 3$, the question as to whether there is a quasi-isometric embedding $\mathrm{H}^{2 n-2} \rightarrow X^{n} \times X^{n}$ remains open. Moreover, the same question is open for quasi-isometric embeddings

$$
\mathrm{H}^{k(n-1)} \rightarrow Y_{k}^{n}, \quad n, k \geq 3 .
$$

Certainly, there is a huge range of possibilities in variation of this theme, e.g., by taking as the target space the product with different dimensions of factors, considering spaces other than $X$, replacing $\mathrm{H}^{p}$ as the source space, etc. However, everything that is known here on the nonembedding side is covered by the ideas of the proof of Theorem 6.7 .

Proof of Theorem 6.7. The key ingredient of the proof is the notion of the hyperbolic dimension of a metric space $X$, hypdim $X$, which was introduced in [BS2] and has the 
following properties:

(1) monotonicity: if $f: X \rightarrow X^{\prime}$ is a quasi-isometric map between metric spaces $X$, $X^{\prime}$, then hypdim $X \leq \operatorname{hypdim} X^{\prime}$;

(2) the product theorem: for any metric spaces $X_{1}, X_{2}$ we have

$$
\operatorname{hypdim}\left(X_{1} \times X_{2}\right) \leq \operatorname{hypdim} X_{1}+\operatorname{hypdim} X_{2} \text {; }
$$

(3) $\operatorname{hypdim} X \leq \operatorname{asdim} X$ for every metric space $X$;

(4) hypdim $\mathbb{R}^{m}=0$ for every $m \geq 0$.

Recall that a metric space $X$ has bounded growth at some scale if for some constants $r, R$ with $R>r>0$ and some $N \in \mathbb{N}$ every ball of radius $R$ in $X$ can be covered by $N$ balls of radius $r$; see [BoS].

By the main result of [BS2], for every geodesic, Gromov hyperbolic space that has bounded growth at some scale and whose boundary at infinity $\partial_{\infty} X$ is infinite, we have

$$
\operatorname{hyp} \operatorname{dim} X \geq \operatorname{dim} \partial_{\infty} X+1 \text {. }
$$

In particular, hypdim $\mathrm{H}^{p} \geq p$ (actually, equality occurs in this case). Now, assume that there is a quasi-isometric embedding $\mathrm{H}^{p} \rightarrow Y_{k}^{n} \times \mathbb{R}^{m}$. Then, by properties (1)-(4) above, we have

$$
p \leq \operatorname{hypdim} \mathrm{H}^{p} \leq k \cdot \operatorname{hypdim} X^{n} \leq k \cdot \operatorname{asdim} X^{n} .
$$

Using Theorem 6.6 and the properties of the asymptotic dimension, or using the fact that $\operatorname{asdim} X^{n} \leq \operatorname{cdim} \partial_{\infty} X^{n}+1$ ([Bu1]) and Theorem 6.3 , we obtain $\operatorname{asdim} X^{n} \leq$ $\operatorname{dim} \partial_{\infty} X^{n}+1=n-1$. Hence, the claim follows.

6.4. Examples of strict inequality in the product theorem for the capacity dimension. An application of Corollary 1.2 is that the strict inequality in the product theorem for the capacity dimension may occur for some compact metric spaces. For each $n \in \mathbb{N}$, consider a Pontryagin surface $\Pi_{n}$, which is a 2-dimensional compact space, $\operatorname{dim} \Pi_{n}=2$; then for coprime $m, n \in \mathbb{N}$ we have $\operatorname{dim}\left(\Pi_{m} \times \Pi_{n}\right)=3$, that is,

$$
\operatorname{dim}\left(\Pi_{m} \times \Pi_{n}\right)<\operatorname{dim} \Pi_{m}+\operatorname{dim} \Pi_{n} .
$$

In accordance with [Dr1], [Dr2, Corollary 2.3], for every prime $p$ there is a hyperbolic Coxeter group with a Pontryagin surface $\Pi_{p}$ as the boundary at infinity. Taken with any visual metric, $\Pi_{p}$ is a locally self-similar space, so that $\operatorname{cdim} \Pi_{p}=\operatorname{dim} \Pi_{p}=2$ by Theorem 1.4. Obviously, the product of locally self-similar spaces is locally self-similar, and we obtain

for prime $p \neq q$.

$$
\operatorname{cdim}\left(\Pi_{p} \times \Pi_{q}\right)<\operatorname{cdim} \Pi_{p}+\operatorname{cdim} \Pi_{q}
$$

Unfortunately, the construction in [Dr2] of an appropriate hyperbolic Coxeter group is implicit. Below, we give explicit constructions of self-similar Pontryagin surfaces.

\section{§7. Self-Similar Pontryagin surfaces}

For the notion of the cohomological dimension of a topological space $X$ with respect to an Abelian group $G, \operatorname{dim}_{G} X$, we refer the reader, e.g., to the survey [Dr3]. Let $p$ be a prime number. A Pontryagin surface $\Pi_{p}$ is a 2-dimensional compact space with $\operatorname{dim}_{\mathbb{Q}} \Pi_{p}=\operatorname{dim}_{\mathbb{Z}_{q}} \Pi_{p}=1$ for every prime $q \neq p$ and $\operatorname{dim}_{\mathbb{Z}_{p}} \Pi_{p}=2$.

Theorem 7.1. For every prime $p$, there exists a Pontryagin surface $\Pi_{p}$ with locally self-similar metric.

Our objective is the existence of a (locally) self-similar metric space $\Pi_{p}$. For a simple argument that proves the required cohomological properties of the compactum $\Pi_{p}$ to be constructed, we refer to [Dr3, Example 1.9]. 
7.1. Construction. By a square we mean a topological space homeomorphic to $[0,1] \times$ $[0,1]$. Given a natural $m \geq 2$, we consider the $m$-band $B_{m}$, which is a 2 -dimensional square complex constructed as follows. The union $\widetilde{B}_{m}$ of $m$ squares with a common side can also be described as $T_{m} \times[0,1]$, where $T_{m}$ is the union of $m$ copies of the segment $[0,1]$ attached to each other along the common vertex 0 . We fix a cyclic permutation $\tau$ of the segments $\sigma \subset T_{m}$ and define $B_{m}=\widetilde{B}_{m} /\left\{\sigma \times 0 \equiv \tau(\sigma) \times 1: \sigma \subset T_{m}\right\}$. If $m=2$, this gives the usual Möbius band.

The square complex $B_{m}$ consists of $m$ squares, and both its boundary $\partial B_{m}$ and its singular locus $s B_{m}$ (corresponding to the common side of the squares) are homeomorphic to $S^{1}$.

For every $a, b>0$, there is a well-defined intrinsic metric on $B_{m}$ with respect to which every square of $B_{m}$ is isometric to the Euclidean rectangle whose sides have length $a$ and $b$. We assume that $a$ is the length of the common side of the squares. Then $\partial B_{m} \subset B_{m}$ is a geodesic of length $m a$ and $s B_{m} \subset B_{m}$ is a geodesic of length $a$. We use the notation $B_{m}(a, b)$ for $B_{m}$ endowed with this metric. Note that $B_{m}(a, b)$ has nonpositive curvature (in the Alexandrov sense) for every $a, b>0$.

Lemma 7.2. Given $a>0, b \geq a \sqrt{2} / 2$, let $A=B_{m}\left(\frac{4 a}{m}, b\right), B=[0, a] \times[0, a]$. Then there is a 1-Lipschitz map

$$
q:(A, \partial A) \rightarrow(B, \partial B)
$$

whose restriction $q \mid \partial A$ to the boundary preserves the length of every arc.

Proof. The boundary $\partial A$ is a closed geodesic in $A$ of length $4 a$. We subdivide $\partial A$ into four segments of length $a$, called the sides, and map $\partial A$ onto $\partial B$ preserving the sides and arc length. This determines $q: \partial A \rightarrow \partial B$, which is obviously 1-Lipschitz.

Next, we extend $q$ to the singular locus $s B_{m}$ by collapsing it to the middle of the square $B$. This extension is still 1-Lipschitz, because the distance between any points $x \in \partial A$ and $x^{\prime} \in s B_{m}$ is at least $b$ and $b \geq a \sqrt{2} / 2$, which is the maximal distance between the middle of $B$ and points of $\partial B$.

Finally, we extend the already defined $q$ to $A$ as the affine map on every radial segment $x x^{\prime} \subset A$, where $x \in \partial A$ and $x^{\prime} \in s B_{m}$ is a unique point closest to $x$. When $x$ runs over $\partial A$, the segments $x x^{\prime}$ cover $A$ so that for different $x, y \in \partial A$, the segments $x x^{\prime}$ and $y y^{\prime}$ have no common interior point. Since $\left|x x^{\prime}\right|=b$, the restriction of $q$ to $x x^{\prime}$ is 1-Lipschitz.

The map $q$ defined in this way is smooth outside of the union of $\partial A, s B_{m}$, and the four radial segments corresponding to the endpoints of the sides of $\partial A$. It is easily seen that $|d q| \leq 1$ there. Since $A$ is geodesic, it follows that $q$ is 1 -Lipschitz.

7.1.1. First template. Fix a natural $m \geq 2$ and an odd $k, k=2 l+1$ with $l \geq 1$. We define a 2-dimensional square complex $P=P_{m, k}$ obtained from the square $Q_{k}=[0, k] \times[0, k]$ by removing the open middle square

$$
q_{k}=(l, l+1) \times(l, l+1) \subset Q_{k}
$$

and attaching instead the $m$-band $B_{m}$ along a homeomorphism $\partial B_{m} \rightarrow \partial q_{k}$. We consider the following square structure on $P$. The remainder $Q_{k} \backslash q_{k}$ consists of $k^{2}-1$ squares, and we subdivide each of them into $m^{2}$ subsquares. Furthermore, the $m$-band consists of $m$ squares, each representable as the rectangle $[0,4] \times[0, m]$ with the natural square structure consisting of $4 m$ squares. We assume that the side $[0,4] \times\{0\}$ corresponds to the singular locus $s B_{m}$ of $B_{m}$. Assuming that the gluing homeomorphism $\partial B_{m} \rightarrow \partial q_{k}$ preserves the induced subdivisions of each circle into $4 \mathrm{~m}$ segments, we obtain the desired square complex structure on $P$ consisting of

$$
s_{m, k}=\left(k^{2}-1\right) m^{2}+4 m^{2}=(k m)^{2}+3 m^{2}
$$


squares. Talking about squares of $P$, we mean this square complex structure. We consider the canonical intrinsic metric on $P$ with respect to which every square of $P$ is isometric to the Euclidean square with the side length $1 /(\mathrm{km})$. The space $P$ is nonpositively curved, the subcomplex $B_{m} \subset P$ is convex and isometric to $B_{m}\left(\frac{4}{k m}, \frac{1}{k}\right)$, and its boundary is geodesic in $P$.

The boundary $\partial P$ consists of four sides of length 1 , and $P$ can be viewed as the unit square $[0,1] \times[0,1]$ with the appropriate middle subsquare replaced by $B_{m}\left(\frac{4}{k m}, \frac{1}{k}\right)$. We have $b>a \sqrt{2} / 2$ for $a=b=1 / k$. Thus, applying Lemma 7.2, we obtain a 1 -Lipschitz map

$$
q_{0}^{1}: P \rightarrow[0,1] \times[0,1],
$$

which is identical outside of the interior of $B_{m} \subset P$.

7.1.2. Constructing a sequence of polyhedra $\left\{P^{i}\right\}$. We construct a sequence of polyhedra $\left\{P^{i}=P_{m, k}^{i}\right\}, i \geq 1$, in such a way that every $P^{i}$ serves as a building block for $P^{i+1}$ and as such is called the $i$ th template. Furthermore, every $P^{i+1}$ consists of one and the same number of building blocks $P^{i}$ independent of $i$. Every polyhedron $P^{i}$ possesses a canonical square complex structure and, being endowed with an intrinsic metric for which every square is isometric to a fixed Euclidean square, is nonpositively curved in the Alexandrov sense. Ideologically, the construction is similar to the well-known constructions of self-similar compact metric spaces via a family of homotheties.

As the 0 th template we take the unit square, $P^{0}=[0,1] \times[0,1]$. The first template $P^{1}=P$ has already been described. It can also be described as follows. The polyhedron $P^{1}$ consists of $s_{m, k}$ blocks, each of which is a $(1 / m k)$-homothetic copy of $P^{0}$ attached along the boundary to the 1-skeleton $S$ of $P^{1}$.

The polyhedron $P^{2}$ is obtained out of $P^{1}$ as follows. We remove each of $s_{m, k}$ open squares of $P^{1}$, obtaining again the 1-dimensional complex $S$, and replace every removed open square by a $(1 / m k)$-homothetic copy of $P^{1}$, attaching it to $S$ along the boundary.

Assume that a square polyhedron $P^{i}$ is already constructed for $i \geq 1$. By assumption, it is endowed with the canonical intrinsic nonpositively curved metric in which every square is isometric to the Euclidean square of side length $1 /(m k)^{i}$. The polyhedron $P^{i}$ consists of $s_{m, k}$ pairwise isometric blocks, each of which is $m k$-homothetic to the template $P^{i-1}$. The boundary $\partial P^{i}$ is subdivided into four sides, each consisting of $(m k)^{i}$ segments of the square structure, and has length 4 .

We construct the square polyhedron $P^{i+1}$ by replacing each of $s_{m, k}$ open squares of $P^{1}$ with a $(1 / m k)$-homothetic copy of the template $P^{i}$ and attaching it to $S$ so that they all together form a square complex structure of $P^{i+1}$ and determine the canonical intrinsic nonpositively curved metric in which every square is isometric to the Euclidean square of side length $1 /(m k)^{i+1}$.

Our construction has the following property: for every integer $j, 1 \leq j \leq i$, the polyhedron $P^{i+1}$ consists of $s_{m, k}^{i+1-j}$ subblocks, each of which is homothetic to the $j$ th template $P^{j}$ with coefficient $(m k)^{i+1-j}$.

Lemma 7.3. The diameter of $P^{i}, i \geq 0$, is bounded above by a constant independent of $i$,

$$
\operatorname{diam} P^{i} \leq d
$$

where one can take $d=d(m, k)=\frac{2 m(l+1)}{m k-1}+2($ recall that $k=2 l+1)$.

Proof. Since the length of the boundary $\partial P^{i}$ equals 4 for every $i \geq 1$, it suffices to estimate $\delta_{i}=\max \left\{\operatorname{dist}\left(x, \partial P^{i}\right): x \in P^{i}\right\}$ from above independently of $i$. 
For $i=1$, the most remote points from the boundary are sitting in the singular locus of the subcomplex $B_{m} \subset P^{1}$. Moving along the 1-skeleton $S$ of $P^{1}$, we see that $\delta_{1}=m / m k+m l / m k=(l+1) / k$.

The grid $S$ serves as a skeleton for attaching the blocks while constructing every $P^{i}$, and thus it is isometrically (in the sense of the induced intrinsic metric) embedded in $P^{i}$ for every $i \geq 1$. So, to estimate $\delta_{i}$ we can use paths in $S$, namely, $\operatorname{dist}_{S}\left(x, S_{0}\right) \leq \delta_{1}$ for every $x \in S$, where $S_{0} \subset S$ is identified with the boundary $\partial P^{i}$ for every $i \geq 1$, and the distance is taken with respect to the intrinsic metric of $S$.

For $i=2$, clearly,

$$
\delta_{2} \leq \delta_{1} / m k+\max _{x \in S} \operatorname{dist}_{S}\left(x, S_{0}\right) \leq \delta_{1} / m k+\delta_{1}
$$

because $P^{2}$ consists of blocks $(1 / m k)$-homothetic to $P^{1}$ whose boundaries are subsets of $S$. Similarly, by recursion we obtain the estimate

$$
\delta_{i+1} \leq \delta_{i} / m k+\delta_{1} \leq \delta_{1} \sum_{j=0}^{i} 1 /(m k)^{j},
$$

which justifies the claim.

7.2. The inverse sequence $\left\{P^{i} ; q_{i}^{i+1}\right\}$. The bonding map $q_{0}^{1}: P^{1} \rightarrow P^{0}$ has already been described above. By induction, we obtain the bonding map $q_{i}^{i+1}: P^{i+1} \rightarrow P^{i}$ for every $i \geq 1$ by putting together the maps $q_{i-1}^{i}$ defined on the blocks of $P^{i+1}$.

This map is 1-Lipschitz, and it is compatible with the self-similar structure of complexes; i.e., its restriction to every subblock $P^{j} \subset P^{i+1}, 1 \leq j \leq i$, coincides with $q_{j-1}^{j}$.

The product $\mathcal{P}=\prod_{i \geq 0} P^{i}$ is the set of all sequences $\left\{x_{i} \in P^{i}: i \geq 0\right\}$. The limit of the inverse sequence $\left\{P^{i} ; q_{i}^{i+1}\right\}$,

$$
\Pi=\Pi_{m, k}=\longleftarrow\left(P^{i} ; q_{i}^{i+1}\right),
$$

is the subset of $\mathcal{P}$ consisting of all sequences $\left\{x_{i}\right\}$ with $x_{i}=q_{i}^{i+1}\left(x_{i+1}\right)$ for every $i \geq 0$. For every $j \geq 0$, we have the projection $q_{j}^{\infty}: \Pi \rightarrow P^{j}$ defined by $q_{j}^{\infty}\left(\left\{x_{i}\right\}\right)=x_{j}$ for every $\left\{x_{i}\right\} \in \Pi$. Clearly, $q_{j}^{j+1} \circ q_{j+1}^{\infty}=q_{j}^{\infty}$ for every $j \geq 0$.

The space $\mathcal{P}$ is compact in the product topology, being the product of compact spaces, and $\Pi$ is closed in $\mathcal{P}$ because all bonding maps are continuous. Thus, $\Pi$ is compact in the induced topology, which we call the product topology of $\Pi$. By the definition of the product topology, the map $q_{j}^{\infty}$ is continuous for every $j \geq 0$, and the product topology is the roughest among all topologies with this property.

For each $\xi=\left\{x_{i}\right\}, \xi^{\prime}=\left\{x_{i}^{\prime}\right\} \in \Pi$, the sequence of distances $\left|x_{i} x_{i}^{\prime}\right|$ is bounded by Lemma 7.3 and monotone nondecreasing because every bonding map is 1-Lipschitz. Now, we define a metric on $\Pi$ by

$$
\left|\xi \xi^{\prime}\right|=\lim _{i}\left|x_{i} x_{i}^{\prime}\right|
$$

(of course, this metric is by no means nonpositively curved despite the fact that all $P^{i}$ are nonpositively curved). We call the corresponding metric topology on $\Pi$ the metric topology of $\Pi$.

Lemma 7.4. The metric topology of $\Pi$ coincides with the product topology.

Proof. If $\left|\xi \xi^{\prime}\right|<r$ for some $\xi=\left\{x_{i}\right\}$ and $\xi^{\prime}=\left\{x_{i}^{\prime}\right\} \in \Pi$, then $\left|x_{i} x_{i}^{\prime}\right| \leq\left|\xi \xi^{\prime}\right|<r$ for every $i \geq 0$. It follows that the projection $q_{i}^{\infty}$ is continuous in the metric topology for every $i \geq 0$, so that every open set in the product topology is open in the metric topology.

Fix $\xi \in \Pi, r>0$ and consider the (open) ball $B_{r}(\xi) \subset \Pi$. We show that there is a subset open in the product topology, contained in $B_{r}(\xi)$, and containing $\xi$. There is 
$j \in \mathbb{N}$ such that $2 d /(m k)^{j}<r$, where $d=d(m, k)$ is the upper bound for the diameter of $P^{i}$ (see Lemma 7.3) and hence for the diameter of $\Pi$.

We consider $x_{j}=q_{j}^{\infty}(\xi) \in P^{j}$ and take the union $\bar{A}$ of all squares of $P^{j}$ containing $x_{j}$. Recall that the side length of every such square is $1 /(m k)^{j}$. Then, the point $x_{j}$ is contained in the interior $A$ of $\bar{A}$, which is open in $P^{j}$. Thus, $B=\left(q_{j}^{\infty}\right)^{-1}(A)$ is open in the product topology, and $\xi \in B$. On the other hand, $\operatorname{diam} B \leq 2 \operatorname{diam} C$, where $C$ is the preimage under $q_{j}^{\infty}$ of a square of $P^{j}$. We have $\operatorname{diam} C=\operatorname{diam} \Pi /(m k)^{j} \leq d /(m k)^{j}$, whence $\operatorname{diam} B \leq 2 d /(m k)^{j}<r$. It follows that $B \subset B_{r}(\xi)$. Therefore, every subset in $\Pi$ open in the metric topology is also open in the product topology.

7.3. Self-similarity of the space $\Pi$. We recall some notions from the theory of selfsimilar metric spaces; see, e.g., [Fa], $[\mathrm{Hu}]$. A compact metric space $K$ is said to be self-similar if there is a finite collection of homotheties $f_{a}: K \rightarrow K, a \in A$, with coefficients $h_{a} \in(0,1)$ such that $K \subset \bigcup_{a \in A} f_{a}(K)$.

In this case, there is a unique number $\mu \geq 0$ with

$$
\sum_{a \in A} h_{a}^{\mu}=1
$$

The number $\mu$ is called the similarity dimension of $K, \mu=\operatorname{dim}_{s} K$ (more precisely, $\mu$ is the similarity dimension of the family $\left.\left\{f_{a}\right\}_{a \in A}\right)$. For example, if $h_{a}=h$ for all $a \in A$, then

$$
\operatorname{dim}_{s} K=\frac{\log |A|}{\log (1 / h)}
$$

Always, $\operatorname{dim}_{H} K \leq \operatorname{dim}_{s} K$ for the Hausdorff dimension $\operatorname{dim}_{H} K$ of $K$. The collection of homotheties $\left\{f_{a}: a \in A\right\}$ satisfies the OSC (Open Set Condition) if there is an open set $U \subset K$ such that $f_{a}(U) \subset U$ for all $a \in A$ and $f_{a}(U) \cap f_{a^{\prime}}(U)=\varnothing$ for all distinct $a, a^{\prime} \in A$. In this case, the Hausdorff dimension of $K$ coincides with the similarity dimension, $\operatorname{dim}_{H} K=\operatorname{dim}_{s} K$ (see, e.g., [EG]).

Proposition 7.5. The metric space $\Pi=\Pi_{m, k}$ is compact and self-similar for every integer $m \geq 2$ and odd $k=2 l+1$. Furthermore, the corresponding collection $\left\{f_{a}: a \in A\right\}$ consists of $|A|=s_{m, k}$ homotheties with coefficients $h_{a}=1 /(m k)$, and satisfies the OSC. In particular, for the Hausdorff dimension we have

$$
\operatorname{dim}_{H} \Pi=2+\frac{\log \left(1+3 / k^{2}\right)}{\log (m k)} .
$$

Proof. Lemma 7.4 implies that the metric space $\Pi$ is compact. Recall that the square polyhedron $P^{i+1}$ consists of $s_{m, k}=(m k)^{2}+3 m^{2}$ blocks with disjoint interiors, each of which is $m k$-homothetic to $P^{i}$ for every $i \geq 0$.

We label the blocks of $P^{i}$ by a finite set $A$ with $|A|=s_{m, k}$ in a way independent of $i$, i.e., compatible with the bonding maps, and, for each $i \geq 0$ and $a \in A$, fix a homothety $f_{a}^{i}: P^{i} \rightarrow P^{i+1}$ with coefficient $h_{a}=1 /(m k)$ whose image is the corresponding block of $P^{i+1}$. We may also assume that

$$
f_{a}^{i} \circ q_{i}^{i+1}=q_{i+1}^{i+2} \circ f_{a}^{i+1}
$$

for each $i \geq 0, a \in A$. Then $P^{i+1}=\bigcup_{a \in A} f_{a}^{i}\left(P^{i}\right)$, and for the interior $U^{i}$ of $P^{i}$ we have $f_{a}^{i}\left(U^{i}\right) \subset U^{i+1}$, while the open sets $f_{a}^{i}\left(U^{i}\right), f_{a^{\prime}}^{i}\left(U^{i}\right)$ are disjoint for different $a, a^{\prime} \in A$.

Relation $(*)$ allows us to pass to the limit as $i \rightarrow \infty$, which yields a collection of homotheties $f_{a}: \Pi \rightarrow \Pi, a \in A$, with coefficients $h_{a}=1 /(m k)$ for every $a \in A$ and with the required properties. 
Proof of Theorem 7.1. We fix an odd $k=2 l+1 \geq 3$ and consider the metric space $\Pi_{p}=\Pi_{p, k}$, which is self-similar by Proposition 7.5. It is an easy exercise to check that every compact self-similar space is locally self-similar. We have $2<\operatorname{dim}_{H} \Pi_{p}<3$ for the Hausdorff dimension by Proposition 7.5; thus, $\operatorname{dim} \Pi_{p} \leq 2$. Applying the argument from [Dr3, Example 1.9], we obtain $\operatorname{dim}_{\mathbb{Q}} \Pi_{p}=\operatorname{dim}_{\mathbb{Z}_{q}} \Pi_{p}=1$ for every prime $q \neq p$ and $\operatorname{dim}_{\mathbb{Z}_{p}} \Pi_{p}=2$. Combined with the estimate $\operatorname{dim} \Pi_{p} \leq 2$, the latter fact implies that $\operatorname{dim} \Pi_{p}=2$.

7.4. Asymptotically self-similar Pontryagin surfaces. We fix a compact self-similar Pontryagin surface $\Pi=\Pi_{m, k}$ for some integer $m \geq 2$ and odd $k=2 l+1 \geq 3$, and define a metric space $\widehat{\Pi}$ as follows. Recall that $\Pi$ consists of $s_{m, k}=(m k)^{2}+3 m^{2}$ blocks, each of which is $(1 / m k)$-homothetic to $\Pi$ and attached along the 1 -skeleton $S$ of the polyhedron $P^{1}$, and $S$ is embedded in $\Pi$ isometrically (in the sense of the induced intrinsic metric). For every $4 m^{2}$ middle blocks, which are projected into the $m$-band $B_{m} \subset P^{1}$, their distance to the boundary $\partial \Pi$ is at least $m l$, because the projection $\Pi \rightarrow P^{1}$ is 1-Lipschitz, and the distance from $B_{m}$ to the boundary $\partial P^{1}$ equals $m l$.

We put $\lambda=m k, X_{0}=\Pi$, and consider the $\lambda$-homothetic copy of $X_{0}, X_{1}=\lambda X_{0}$. The space $X_{1}$ consists of $s_{m, k}$ blocks isometric to $X_{0}$, and we can view $X_{0}$ as a subspace of $X_{1}, X_{0} \subset X_{1}$, identifying it with some block. Moreover, we take this block in the middle of $X_{1}$ so that $\operatorname{dist}\left(X_{0}, \partial X_{1}\right) \geq \lambda m l$. Taking $X_{i}=\lambda^{i} X_{0}$, we obtain an increasing sequence

$$
X_{0} \subset X_{1} \subset \cdots \subset X_{i}
$$

of metric spaces via appropriate identifications for which $\operatorname{dist}\left(X_{i}, \partial X_{i+1}\right) \geq \lambda^{i+1} m l$. Now, we define $\widehat{\Pi}=\widehat{\Pi}_{m, k}:=\bigcup_{i \in \mathbb{N}} X_{i}$. Given $x, x^{\prime} \in \widehat{\Pi}$, there is $i \in \mathbb{N}$ with $x, x^{\prime} \in X_{i}$. Then, the distance $\left|x x^{\prime}\right|$ in $\widehat{\Pi}$ is well defined as the distance between $x, x^{\prime}$ in $X_{i}$. Therefore, $\widehat{\Pi}$ is a metric space.

Proposition 7.6. The metric space $\widehat{\Pi}$ is asymptotically similar to the compact space $\Pi$.

Proof. We put $\lambda=\Lambda_{0}=m k$ and consider a bounded subset $A \subset \widehat{\Pi}$ with $\operatorname{diam} A \leq R / \Lambda_{0}$ for some $R>1$. There is $i \in \mathbb{N}$ with $\lambda^{i}<R \leq \lambda^{i+1}$. Then, any $\lambda^{i}$-homothety and $\lambda^{i+1}$-homothety is a $\lambda$-quasihomothety with coefficient $R$.

Since $\operatorname{dist}\left(X_{j}, \partial X_{j+1}\right) \rightarrow \infty$ as $j \rightarrow \infty, A$ is contained in some $X_{j+1}$, and we may assume that $j \geq i$. The problem is, however, that $j$ can be much larger than $i$, e.g., if $A$ is sitting near the boundary of $X_{j}$. Thus, we assume that $j \geq i$ is minimal with the property that $A^{\prime} \subset X_{j+1}$ for some isometric copy $A^{\prime} \subset \widehat{\Pi}$ of $A$. Now, we show that $j \leq i+1$.

Suppose the contrary; then $j \geq i+2$. Note that $\lambda=m k \geq 6$. Then $R / \Lambda_{0} \leq \lambda^{i} \leq$ $\lambda^{i+1} / 6$. The space $X_{j+1}$ is the union of $s_{m, k}^{2}$ subblocks, each of which is isometric to $X_{j-1}$. Since $j-1 \geq i+1$ and the distance in $X_{j+1}$ between any disjoint copies of $X_{j-1}$ is at least $\lambda^{i+1}$, we see that $A$ is covered by the union $Q \subset X_{j+1}$ of copies of $X_{j-1}$ such that there is a common point of the copies. However, every such union is isometric to a subset of $X_{j}$. Hence, $X_{j}$ contains an isometric copy of $A$. Since this contradicts our assumption on $j$, we conclude that $j \leq i+1$.

It follows that the $\lambda^{j+1}$-homothety $f: \Pi \rightarrow X_{j+1} \subset \widehat{\Pi}$ is a $\lambda$-quasihomothety with coefficient $R$, and its image $X_{j+1}$ contains an isometric copy of $A$. Therefore, $\widehat{\Pi}$ is asymptotically similar to $\Pi$.

Using Theorem 1.3, we obtain $\operatorname{asdim} \widehat{\Pi}=\operatorname{dim} \Pi=2$. We fix an odd $k=2 l+1 \geq 3$ and put $\widehat{\Pi}_{p}=\widehat{\Pi}_{p, k}$. 
Corollary 7.7. For distinct primes $p$, $q$ we have

$$
\operatorname{asdim}\left(\widehat{\Pi}_{p} \times \widehat{\Pi}_{q}\right)<\operatorname{asdim} \widehat{\Pi}_{p}+\operatorname{asdim} \widehat{\Pi}_{q} .
$$

Proof. If metric spaces $X, X^{\prime}$ are asymptotically similar to metric spaces $Y, Y^{\prime}$, respectively, then clearly $X \times X^{\prime}$ is asymptotically similar to $Y \times Y^{\prime}$. Hence, $\operatorname{asdim}\left(\widehat{\Pi}_{p} \times \widehat{\Pi}_{q}\right)$ $=\operatorname{dim}\left(\Pi_{p} \times \Pi_{q}\right)=3$, while $\operatorname{asdim} \widehat{\Pi}_{p}=\operatorname{dim} \Pi_{p}=2$ and $\operatorname{asdim} \widehat{\Pi}_{q}=\operatorname{dim} \Pi_{q}=2$.

Remark 7.8. In [Gra], examples of coarse spaces $X, Y$ with $\operatorname{asdim}(X \times Y)<\operatorname{asdim} X+$ $\operatorname{asdim} Y$ were given. There, the asymptotic dimension $\operatorname{asdim} X$ is associated with the coarse structure $\mathcal{E}$ of $X$ and it would be more appropriate to use the notation $\mathcal{E} \operatorname{dim} X$ for that dimension. The spaces $X, Y$ are of the form $K \times[0,1)$ where $K$ is a classical Pontryagin surface, that is, a 2-dimensional compact space with $\operatorname{dim}_{\mathbb{Q}} K=\operatorname{dim}_{\mathbb{Z}_{q}} K=1$ for every prime $q \neq p$ and $\operatorname{dim}_{\mathbb{Z}_{p}} K=2$ for some prime $p$. The coarse structure on $X=$ $K \times[0,1)$ is the topological coarse structure (in terms of the book [Ro]) or the continuously controlled coarse structure (in terms of [Gra]) induced by the compactification $\bar{X}=$ $K \times[0,1]$ of $X$, and $\bar{X}$ is certainly metrizable.

We want to explain that these examples do not cover the case of the classical asymptotic dimension, introduced in $[\mathrm{Gr}]$, for which we have constructed our asymptotically self-similar Pontryagin surfaces. The usual asymptotic dimension of a metric space $(X, d)$ is associated with the bounded coarse structure $\mathcal{E}_{d}$, asdim $X=\mathcal{E}_{d} \operatorname{dim} X$, where a set $E \subset X \times X$ is controlled, $E \in \mathcal{E}_{d}$, if and only if $\sup \left\{d\left(x, x^{\prime}\right):\left(x, x^{\prime}\right) \in E\right\}$ is finite. However, it is known that the coarse structure induced by any metrizable compactification is nonmetrizable; see [Ro, Example 2.53 and Remark 2.54]. In particular, the coarse structure of $X=K \times[0,1)$ above is nonmetrizable; i.e., there is no metric $d$ on $X$ for which $\mathcal{E}=\mathcal{E}_{d}$, and therefore, it does not make sense to compare $\mathcal{E} \operatorname{dim} X$ with the classical asymptotic dimension. Rather, the arguments in [Gra, Theorem 2.5.7] show that $\mathcal{E} \operatorname{dim} X=\operatorname{dim} K+1$ coincides with the topological dimension of $X, \mathcal{E} \operatorname{dim} X=\operatorname{dim} X$.

\section{ACKNOWLEDGEMENTS}

The first author is grateful to Bruce Kleiner and Viktor Schroeder for numerous stimulating discussions related to this paper. We are also grateful to Alexander Dranishnikov for informing us about the paper [Sw].

\section{REFERENCES}

[Al] P. Alexandroff, Dimensionstheorie. Ein Beitrag zur Geometrie der abgeschlossenen Mengen, Math. Ann. 106 (1932), 161-238.

[As] P. Assouad, Plongements lipschitziens dans $R^{n}$, Bull. Soc. Math. France 111 (1983), $429-448$. MR0763553 (86f:54050)

[BD] G. Bell and A. Dranishnikov, On asymptotic dimension of groups, Algebr. Geom. Topol. 1 (2001), 57-71. MR1808331 (2001m:20062)

[BoS] M. Bonk and O. Schramm, Embeddings of Gromov hyperbolic spaces, Geom. Funct. Anal. 10 (2000), no. 2, 266-306. MR1771428 (2001g:53077)

[Bou] M. Bourdon, Immeubles hyperboliques, dimension conforme et rigidité de Mostow, Geom. Funct. Anal. 7 (1997), 245-268. MR1445387 (98c:20056)

[BF] N. Brady and B. Farb, Filling-invariants at infinity for manifolds of nonpositive curvature, Trans. Amer. Math. Soc. 350 (1998), 3393-3405. MR1608281 (99c:53039)

[Bu1] S. V. Buyalo, Asymptotic dimension of a hyperbolic space and the capacity dimension of its boundary at infinity, Algebra i Analiz 17 (2005), no. 2, 70-95; English transl., St. Petersburg Math. J. 17 (2006), no. 2, 267-283; arXive:math.GT/0505427. MR2159584 (2006d:31009)

[Bu2] _ Capacity dimension and embedding of hyperbolic spaces into a product of trees, Algebra $\mathrm{i}$ Analiz 17 (2005), no. 4, 42-58; English transl., St. Petersburg Math. J. 17 (2006), no. 4, 581-591; arXive:math.GT/0505429. MR2173936 (2006e:31008)

[Bu3] - Volume entropy of hyperbolic graph surfaces, Ergodic Theory Dynam. Systems 25 (2005), 403-417. MR2129103 (2006a:37021) 
[BS1] S. Buyalo and V. Schroeder, Embedding of hyperbolic spaces in the product of trees, Geom. Dedicata 113 (2005), 75-93; arXive:math. GT/0311524 (2003). MR2171299 (2006f:53055)

[BS2] _ Hyperbolic dimension of metric spaces, Algebra i Analiz 19 (2007), no. 1, 93-108; English transl. in St. Petersburg Math. J. 19 (2008), no. 1. MR2319511

[Dr1] A. Dranishnikov, On the virtual cohomological dimensions of Coxeter groups, Proc. Amer. Math. Soc. 125 (1997), 1885-1891. MR1422863 (98d:55001)

[Dr2] - Boundaries of Coxeter groups and simplicial complexes with given links, J. Pure Appl. Algebra 137 (1999), 139-151. MR1684267 (2000d:20069)

[Dr3] Cohomological dimension theory of compact metric spaces, Topology Atlas Invited Contributions 6 (2001), no. 1, 7-73.

[DS] A. Dranishnikov and V. Schroeder, Embedding of hyperbolic Coxeter groups into products of binary trees and aperiodic tilings, arXive:math. GT/0504566 (2005).

[DZ] A. Dranishnikov and M. Zarichnyi, Universal spaces for asymptotic dimension, Topology Appl. 140 (2004), 203-225. MR2074917 (2005e:54032)

[EG] G. A. Edgar and J. Golds, A fractal dimension estimate for a graph-directed iterated function system of non-similarities, Indiana Univ. Math. J. 48 (1999), 429-447. MR1722803 (2001b:28012)

[Fa] K. Falconer, Techniques in fractal geometry, John Wiley and Sons, Chichester, 1997. MR1449135 (99f:28013)

[Gra] B. Grave, Asymptotic dimension of coarse spaces, Preprint, 2004.

[Gr] M. Gromov, Asymptotic invariants of infinite groups, Geometric Group Theory, Vol. 2 (Sussex, 1991) (G. A. Niblo, M. A. Roller, eds.), London Math. Soc. Lecture Note Ser., vol. 182, Cambridge Univ. Press, Cambridge, 1993, pp. 1-295. MR1253544 (95m:20041)

[Hu] J. Hutchinson, Fractals and self-similarity, Indiana Univ. Math. J. 30 (1981), $713-747$. MR0625600 (82h:49026)

[LS] U. Lang and T. Schlichenmaier, Nagata dimension, quasisymmetric embeddings, and Lipschitz extensions, Int. Math. Res. Not. 2005, no. 58, 3625-3655; arXive:math. MG/0410048 (2004). MR2200122 (2006m:53061)

[Ro] J. Roe, Lectures on coarse geometry, Univ. Lecture Ser., vol. 31, Amer. Math. Soc., Providence, RI, 2003. MR2007488 (2004g:53050)

[Sw] J. Swiatkowski, On the asymptotic homological dimension of hyperbolic groups, Bull. London Math. Soc. 27 (1995), 209-221. MR1328695 (96c:20070)

St. Petersburg Branch, Steklov Mathematical Institute, Russian Academy of Sciences, Fontanka 27, St. Petersburg 191023, Russia

E-mail address: sbuyalo@pdmi.ras.ru

St. Petersburg Branch, Steklov Mathematical Institute, Russian Academy of Sciences, Fontanka 27, St. Petersburg 191023, Russia

E-mail address: lebed@pdmi.ras.ru

Received 29/SEP/2005

Translated by S. BUYALO 